\title{
EVALUATING MODULAR FORMS ON SHIMURA CURVES
}

\author{
PAUL D. NELSON
}

\begin{abstract}
Let $f$ be a newform, as specified by its Hecke eigenvalues, on a Shimura curve $X$. We describe a method for evaluating $f$. The most interesting case is when $X$ arises as a compact quotient of the hyperbolic plane, so that classical $q$-expansions are not available. The method takes the form of an explicit, rapidly-convergent formula that is well-suited for numerical computation. We apply it to the problem of computing modular parametrizations of elliptic curves, and illustrate with some numerical examples.

An important ingredient is a new method for numerically computing Petersson inner products, which may be of independent interest.
\end{abstract}

\section{INTRODUCTION}

1.1. Computing modular parametrizations. Let $E$ be an elliptic curve over a totally real number field $F$. By a modular parametrization of $E$, we mean a surjective morphism

$$
\operatorname{Jac} X_{B} \rightarrow E
$$

of abelian varieties over $F$, where $X_{B}$ is the Shimura curve attached to an order in a quaternion $F$-algebra $B$ (see $\S \S 2.2$ and 2.3). The images under (1) of certain special divisors on $X_{B}$ are called Heegner points (see 84.1 ), and provide some of the most versatile examples of analytic constructions of solutions to algebraic equations (see e.g. 8, 10, 15, 17, 40,).

It is of interest to compute (numerically) the curves $X_{B}$, the maps (1), and the images thereunder of special divisors. Methods for doing so have been given in many, but not all, cases of interest. When $X_{B}$ has very low genus (e.g., genus 0), one can work with an explicit algebraic description of $X_{B}$ (see [9, 36]); in general, such a model becomes unmanageable and one must resort to analytic means (see [5. §3.2]). Methods for computing Heegner points using $p$-adic analysis (see [4, 12] and the references therein) apply when $B$ ramifies at some finite place, but there are cases of interest in which $B$ ramifies at no finite place, such as when $F \neq \mathbb{Q}$ has odd degree and $E$ is everywhere unramified; in that setting, Darmon-Rotger [5, §3.2] describe as "completely open" the problem of computing Heegner points. Even when $p$-adic methods apply, it is of independent interest, and described as an open problem by Greenberg [13, p. 5], to obtain a uniform archimedean-analytic method for computing Heegner points.

We are motivated by the latter problem of computing Heegner points, and more generally modular parametrizations (1), using archimedean analysis.

Received by the editor October 9, 2012 and, in revised form, October 10, 2013.

2010 Mathematics Subject Classification. Primary 11F11, 11Y40; Secondary 11F27.

The author was supported by NSF grant OISE-1064866 and partially supported by grant SNF-137488 during the completion of this paper. 
1.2. Evaluating modular forms. It is classical that the map (1) is given by a certain holomorphic differential $f_{B}(z) d z$ on $X_{B}$, in the sense that the composition

$$
\operatorname{Pic}^{0} X_{B}(\mathbb{C}) \cong \operatorname{Jac} X_{B}(\mathbb{C}) \rightarrow E(\mathbb{C}) \cong \mathbb{C} / \Lambda
$$

of the Abel-Jacobi map for $X_{B}$ with the inverse Weierstrass parametrization of $E$ should agree, up to isogeny, with the linear extension of

$$
\left[\tau_{1}\right]-\left[\tau_{0}\right] \mapsto \int_{\tau_{0}}^{\tau_{1}} f_{B}(z) d z
$$

A fundamental result of Eichler-Shimura (see [31, §7.4], [40, §1.4.9], [3, §10.3]) characterizes the weight two newform $f_{B}$ (up to a normalizing scalar) by expressing its Hecke eigenvalues in terms of the arithmetic of $E$ over finite fields.

Thus the problem of computing modular parametrizations reduces to that of evaluating a newform of known Hecke eigenvalues on a Shimura curve. This problem is of independent interest. For example, near the end of his paper Shimura Curve Computations, Elkies [9, §5.5] writes:

The reader will note that so far we have said nothing about computing with modular forms on Shimura curves. Not only is this an intriguing question in its own right, but solving it may also allow more efficient computation of Shimura curves and the natural maps between them, as happens in the classical modular setting.

We distinguish three senses in which one can "compute" the space of holomorphic modular forms of given positive even integral weight on a Shimura curve $X_{B}$ :

(I) as an abstract vector space.

(II) as an abstract Hecke module.

(III) as a concrete Hecke module realized as a space of differentials on $X_{B}$.

Solutions to problem (I) are given by classical dimension formulas [31, §2.6] and to problem (II) by some classical algorithms involving Brandt modules [27], modular symbols [32, and their generalizations (see [6, 7, 14, 18, 38] and the references therein). In this paper, we address the passage from a known solution of problem (II) to one of problem (III).

1.3. The role of Fourier expansions. If $F=\mathbb{Q}$ is the rational number field, $B=M_{2}(\mathbb{Q})$ is the matrix algebra, and (for simplicity) $X_{M_{2}(\mathbb{Q})}=X_{0}(N)$ is the usual compactification of $\Gamma_{0}(N) \backslash \mathbb{H}$ for some natural number $N$, then $X_{M_{2}(\mathbb{Q})}$ has the cusp $\infty$ at which $f=f_{M_{2}(\mathbb{Q})}$ admits a Fourier expansion

$$
f(z)=\sum_{n \in \mathbb{N}} a_{f}(n) q^{n} \quad \text { with } q:=e(z):=e^{2 \pi i z}, i=\sqrt{-1}
$$

for some complex coefficients $a_{f}(n)$, where $a_{f}(1)$ is nonzero and explicitly normalized. The ratios $a_{f}(n) / a_{f}(1)$, which depend multiplicatively on $n$, are determined explicitly by the Hecke eigenvalues of $f$, and hence, via the Eichler-Shimura relations, by computable invariants of $E$. For example, if $p$ is a prime not dividing $N$, then $a_{f}(p) / a_{f}(1)=p+1-\# E\left(\mathbb{F}_{p}\right)$. Thus one may compute the map $\operatorname{Jac} X_{M_{2}(\mathbb{Q})} \rightarrow E$ by summing the series

$$
\int_{\tau_{0}}^{\tau_{1}} f(z) d z=\frac{1}{2 \pi i} \sum_{n \in \mathbb{N}} \frac{a_{f}(n)}{n}\left(e\left(n \tau_{1}\right)-e\left(n \tau_{0}\right)\right)
$$

obtained by integrating (2) term-by-term (see e.g. [8,23]) 1

\footnotetext{
${ }^{1}$ We suppress here discussion of certain technicalities, such as the choice of cusp at which to expand $f$.
} 
If $B \neq M_{2}(\mathbb{Q})$, then $X_{B}$ has no cusps (equivalently, the group $\Gamma$ has no parabolic elements), so $f_{B}$ does not admit an expansion of the type (2). The absence of such an expansion makes it more difficult to evaluate $f_{B}$; in fact, we are not aware of any successful attempts to do so prior to the work described in this paper.

1.4. Summary of results. We will describe

(A) a general method for computing the values and line integrals of modular forms (and their derivatives) on Shimura curves $X_{B}$ using only archimedean analysis (see \$5), and

(B) an explicit, "ready-to-use" formula, obtained by applying the method (A), in the simple but already interesting case that $B$ is an indefinite quaternion algebra over $\mathbb{Q}$ and $X_{B}$ is attached to an Eichler order of squarefree level (see 93 ).

We apply the formula (B) to several numerical examples in $\$ 4$ so that $\S \S 3$, 4 and 5 follow roughly a "theorem-application-proof" paradigm. Applications of the method (A) to other settings, such as those arising from Shimura curves attached to quaternion algebras over totally real fields, will be taken up in a future paper. The method itself is general, and extends to arbitrary automorphic quotients attached to unit groups of quaternion algebras over number fields.

Explicit formula. To sidestep the notational preliminaries needed to state formula (B) precisely, we illustrate it here in the simplest nontrivial case. Let $\Delta(z):=$ $q \prod_{n \in \mathbb{N}}\left(1-q^{n}\right)^{24}=\sum_{n \in \mathbb{N}} a_{\Delta}(n) q^{n}$ be the Ramanujan/discriminant function on the upper half-plane $\mathbb{H}:=\{z=x+i y: y>0\}$. It satisfies the functional equation $\Delta(-1 / z)=z^{12} \Delta(z)$ and spans the space of cusp forms of weight 12 on $\mathrm{SL}_{2}(\mathbb{Z})$. A famous theorem of Deligne implies that $\left|a_{\Delta}(n)\right| \leq n^{11 / 2} \tau(n)$ with $\tau(n)$ the number of positive divisors of $n$.

One can evaluate $\Delta$ on the positive imaginary axis $z=i y$ by summing the series

$$
\Delta(i y)=\sum_{n \in \mathbb{Z}} a_{\Delta}(n) e^{-2 \pi n y},
$$

which converges rapidly provided that $y$ is not too small 2 A very special case of Theorem 3.1 is a new formula for $\Delta(i y)$, which, while visibly "worse" than (3), has the virtue of extending to modular forms on compact Shimura curves for which analogues of (3) are not available:

Theorem 1.1. Let $y_{1}$ and $y_{2}$ be positive reals. Then

$$
\left(y_{1} y_{2}\right)^{6} \Delta\left(i y_{1}\right) \Delta\left(i y_{2}\right)=\sum_{\substack{a, b, c, d \in \mathbb{Z} \\ a d-b c>0}} a_{\Delta}(a d-b c) e^{12 i \theta} W_{12}(2 \pi r),
$$

where $r \in \mathbb{R}_{+}^{\times}$and $\theta \in \mathbb{R} / 2 \pi \mathbb{Z}$ are the polar coordinates defined by

$$
r e^{i \theta}=a \sqrt{y_{2} / y_{1}}+d \sqrt{y_{1} / y_{2}}+i\left(b / \sqrt{y_{1} y_{2}}-c \sqrt{y_{1} y_{2}}\right) \in \mathbb{C}^{\times}
$$

and $W_{12}$ is the rapidly-convergent infinite sum of Bessel functions

$$
W_{12}(x)=2^{-11} \sum_{n \in \mathbb{N}} n^{12}\left(n x K_{11}(n x)-K_{12}(n x)\right) .
$$

\footnotetext{
${ }^{2}$ The latter condition is not serious, as the functional equation $\Delta(i / y)=y^{12} \Delta(i y)$ allows one to assume that $y \geq 1$.

${ }^{3}$ For notational simplicity, we suppress the dependence of $r$ and $\theta$ on $y_{1}, y_{2}, a, b, c, d$.
} 


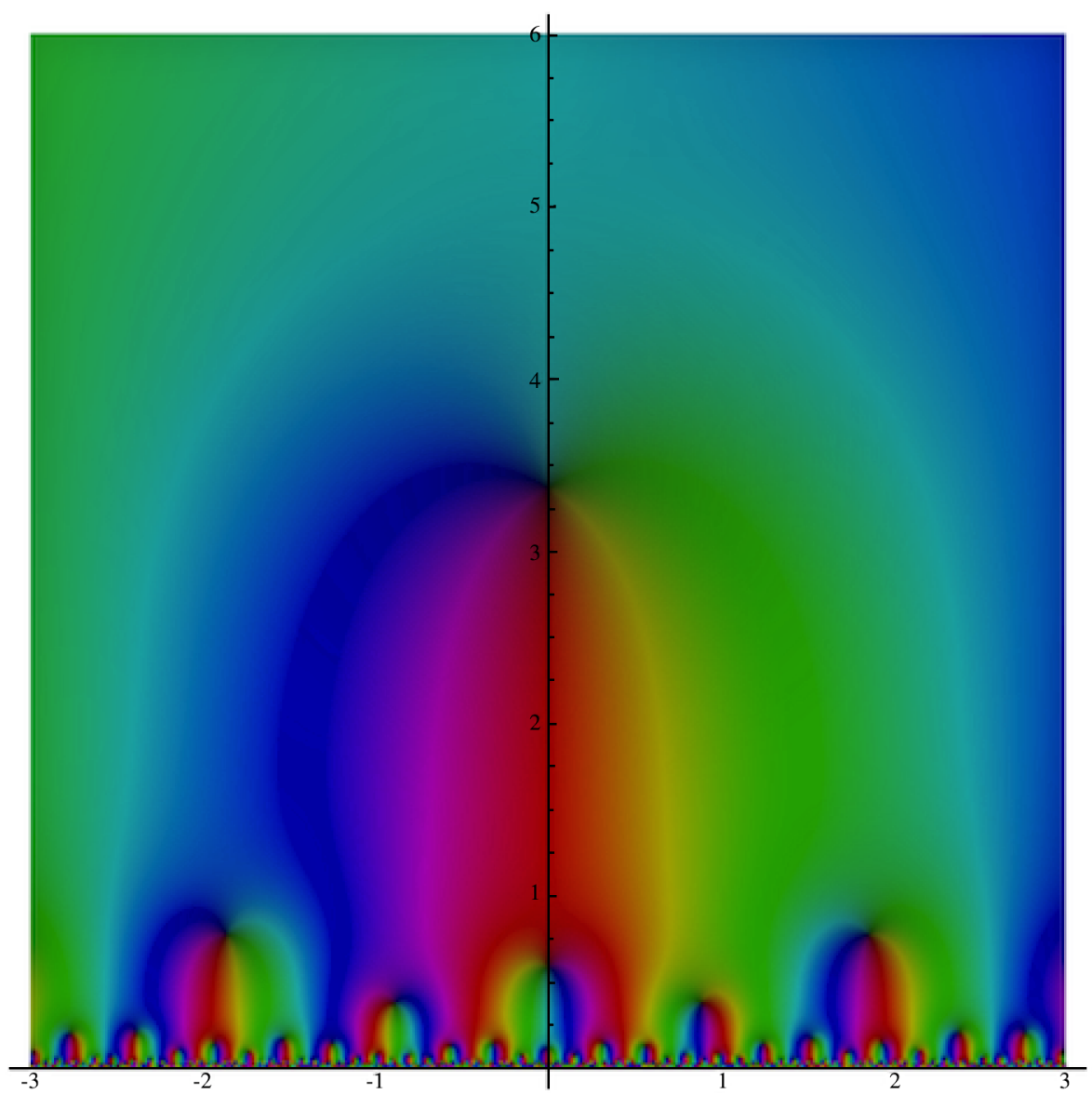

Figure 1. The "first" modular form on a compact Shimura curve: $\operatorname{Im}(z)^{4 / 2} f(z)$ for $f: \mathbb{H} \rightarrow \mathbb{C}$ in the one-dimensional space $S_{4}\left(\Gamma_{0}^{6}(1)\right)$, as depicted by SAGE's complex_plot command and evaluated via the method of this paper. Here $\Gamma_{0}^{6}(1)=\left(\sum_{i=1}^{4} \mathbb{Z} e_{i}\right) \cap$ $\mathrm{SL}_{2}(\mathbb{R})$, where $e_{1}=[1,0 ; 0,1], e_{2}=[1 / \sqrt{2}, 1 / \sqrt{2} ; 3 / \sqrt{2},-1 / \sqrt{2}]$, $e_{3}=[1 / 2,1 / 2 ;-3 / 2,1 / 2]$, and $e_{4}=[0, \sqrt{2} ; 3 \sqrt{2}, 0]$.

It is true, although not completely obvious, that the RHS of (4) converges absolutely, and in fact, rapidly; see Remark 3.3

The general formula given by Theorem 3.1 is no less explicit in principle than that of Theorem 1.1. It follows that one can compute modular parametrizations by compact Shimura curves in terms of integrals of expressions essentially of the shape (4) (see \$4 for numerical examples).

In Theorem 3.9, we give formulas for the values of Shimura-Maass derivatives of newforms, which may be used to compute Taylor expansions with respect to suitable local parameters. Such formulas should provide an effective tool for computing equations of Shimura curves, but we do not pursue such applications here.

The formula (41) is similar to those arising from standard methods for computing values of $L$-functions, so one interpretation of the results of this paper is that it 
is no more difficult to evaluate automorphic forms on quaternion algebras than to evaluate $L$-functions of logarithmically-comparable conductor.

General method. We turn to sketching the general method (A), of which a more detailed account will be found in \$5. Recall, from \$1.2, that the problem under consideration is to recover the values of a newform of given Hecke eigenvalues on a Shimura curve (see $₫ 2$ for definitions). The abstract possibility of such recovery is the content of the multiplicity one theorem (henceforth abbreviated M1) of Jacquet-

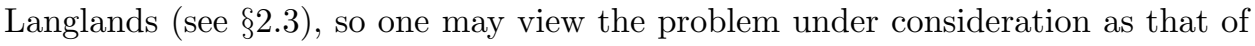
giving a computationally effective realization of M1. From this perspective, our main tool suggests itself naturally, as we now explain.

Jacquet-Langlands proved M1 by establishing a correspondence between automorphic forms on $B^{\times}$and on $\mathrm{GL}_{2}$, identifying scaling classes of newforms on compact Shimura curves over $\mathbb{Q}$ with those on congruence covers of $\mathrm{SL}_{2}(\mathbb{Z}) \backslash \mathbb{H}$. Their proof involved a comparison of instances of the Selberg trace formula, and was nonconstructive.

Shimizu [30], generalizing earlier work of Eichler, realized the Jacquet-Langlands correspondence analytically as a theta correspondence between $\mathrm{GO}(B)$ and $\mathrm{GL}_{2}$. Since $\mathrm{GL}_{2}$ forms admit Fourier expansions, Shimizu's result reduces our task to making that theta correspondence computationally effective.

A new key ingredient, which may be of independent computational and theoretical interest, is a technique for computing Petersson inner products on finite index subgroups of $\mathrm{SL}_{2}(\mathbb{Z})$ that does not require knowledge of an explicit fundamental domain (see 95.3 ). We have in mind the natural generalization to the setting of automorphic forms on higher-dimensional quotients attached to $\mathrm{GL}_{2}$ over a number field, where explicit fundamental domains (and multidimensional oscillating integrals thereon) seem prohibitively complicated.

1.5. Other approaches. A precursor to the method of this paper was implemented in January 2011, described in the notes [26, and presented at the Arizona Winter School in March 2011. A preprint of Voight and Willis 35] describes another technique, quite different from our own, for performing computations on compact Shimura curves. Inspired by methods of Stark and Hejhal for computing Fourier expansions of Maass forms on $\mathrm{SL}_{2}(\mathbb{Z})$, the authors compute the Taylor expansion of a modular form on a compact Shimura curve in a suitable local parameter by solving for the linear conditions imposed by the automorphy and Hecke relations. Both techniques seem worth developing; for example, while the approach described by Voight and Willis is sufficiently flexible to extend even to noncongruence or nonarithmetic Fuchsian groups, the method described in this paper seems strong for certain applications, such as computing modular parametrizations of elliptic curves, in which there are already well-developed methods for obtaining the Hecke eigenvalues. We thank John Voight for several lively discussions of such matters.

\section{BACKGROUND AND NOTATION}

2.1. Summary. The remaining subsections (\$2.2 92.4 of $₫ 2$ aim to make precise the summary below, and may be skimmed or referred to as necessary. 
Groups. For each squarefree integer $D$ possessing an even number of prime factors, and for each positive integer $N$ coprime to $D$, we fix a Fuchsian group

$$
\Gamma_{0}^{D}(N)=R \cap \mathrm{SL}_{2}(\mathbb{R})<\mathrm{SL}_{2}(\mathbb{R})
$$

arising from a choice of Eichler order $R$ of level $N$ in a quaternion algebra $B / \mathbb{Q}$ of discriminant $D$ (see $\$ 2.2$ ) together with a sequence of inclusions

$$
R \subset B \hookrightarrow M_{2}(\mathbb{R})
$$

(see Definition 2.1). We take $\Gamma_{0}^{1}(N)=\Gamma_{0}(N)$ for convenience.

Newforms. For each $\Gamma<\mathrm{SL}_{2}(\mathbb{R})$ as above and each positive even integer $k$, we define the set $\mathcal{F}_{k}(\Gamma)$ of (holomorphic cuspidal) newforms on $\Gamma$ (see \$2.3).

Jacquet-Langlands correspondents. Let $\Gamma^{\prime}=\Gamma_{0}^{D}(N)$ and $\Gamma=\Gamma_{0}(D N)$. We recall the Jacquet-Langlands correspondence as a natural bijection $\mathcal{F}_{k}\left(\Gamma^{\prime}\right) / \mathbb{C}^{\times}=$ $\mathcal{F}_{k}(\Gamma) / \mathbb{C}^{\times}$between scaling classes of newforms (see $\left.\$ 2.4\right)$. We say that a pair of newforms $f^{\prime} \in \mathcal{F}_{k}\left(\Gamma^{\prime}\right), f \in \mathcal{F}_{k}(\Gamma)$ corresponding thereunder are compatibly normalized if $f(z)=\sum_{n \in \mathbb{N}} a_{f}(n) e(n z)$ with $a_{f}(1)=1$ and the Petersson norms of $f$ and $f^{\prime}$ with respect to standard hyperbolic measures coincide (see Definition 2.2).

2.2. Quaternion algebras and Eichler orders. We collect here some background on quaternion algebras and Eichler orders that will be relevant for what follows. We refer to 34 for details and proofs.

Quaternion algebras over general fields. Let $F$ be a field. A quaternion algebra $B$ over $F$ is a simple $F$-algebra with center $F$ and dimension $2^{2}=4$ over $F$. A basic example is the algebra $M_{2}(F)$ of $2 \times 2$ matrices. A quaternion algebra is called split, or said to split, if it is isomorphic to $M_{2}(F)$ (as an $F$-algebra); it is called nonsplit, or said not to split, otherwise. For $a, b \in F^{*}$, let $B=(a, b \mid F)$ denote the $F$-algebra $F \oplus F i \oplus F j \oplus F i j$, where $i, j$ satisfy the relations $i^{2}=a, j^{2}=b$, and $i j=-j i$. Then $(a, b \mid F)$ is a quaternion algebra over $F$, and every quaternion algebra over $F$ is isomorphic to one of this form provided that $F$ is not of characteristic 2 .

Let $E$ be an extension field of $F$. The most relevant examples are quadratic field extensions of $F$, and completions of $F$ if $F$ is a number field. Say that $E$ splits $B$, or that $B$ splits at/over $E$, etc., if $B \otimes_{F} E \cong M_{2}(E)$ splits. It is known that $E$ splits $B$ if and only if there exists an embedding $B \hookrightarrow M_{2}(E)$ (of $F$-algebras). Every quaternion algebra over $F$ is split by some quadratic field extension of $F$. For example, if $a$ is not a square in $F$, then $F(\sqrt{a})$ splits $(a, b \mid F)$, and we have an embedding

$$
(a, b \mid F) \ni x_{0}+x_{1} i+x_{2} j+x_{3} i j \mapsto\left[\begin{array}{cc}
x_{0}+x_{1} \sqrt{a} & x_{2}+x_{3} \sqrt{a} \\
b\left(x_{2}-x_{3} \sqrt{a}\right) & x_{0}-x_{1} \sqrt{a}
\end{array}\right] \in M_{2}(F(\sqrt{a})) .
$$

A quaternion algebra $B$ comes equipped with a main involution $\iota$ that induces the nontrivial automorphism of every quadratic field extension of $F$ contained in $B$. If $B \hookrightarrow M_{2}(E)$ as above, then $\iota$ may be obtained by restricting the adjoint map $\left(\begin{array}{ll}a & b \\ c & d\end{array}\right) \mapsto\left(\begin{array}{cc}d & -b \\ -c & a\end{array}\right)$. We denote by

$$
\begin{aligned}
\operatorname{det}(\alpha) & =\alpha \alpha^{\iota} \in F, \\
\operatorname{tr}(\alpha) & =\alpha+\alpha^{\iota} \in F,
\end{aligned}
$$


the (reduced) norm and (reduced) trace on $B 4$ The trace induces an $F$-valued bilinear form

$$
\left\langle b_{1}, b_{2}\right\rangle=\operatorname{tr}\left(b_{1} b_{2}^{\iota}\right)=b_{1} b_{2}^{\iota}+b_{1}^{\iota} b_{2}
$$

on $B$, called the trace pairing.

Quaternion algebras over number fields. Suppose henceforth that $F$ is a number field, and let $|F|$ be its set of places. If $v \in|F|$, say that $v$ splits $B$, or that $B$ splits at/over $v$, if $F_{v}$ splits $B$. For example, if $a, b \in F^{\times}$, then $(a, b \mid F)$ splits at $v$ if and only if the Hilbert symbol $(a, b)_{v}$, which is defined to be 1 if $a$ is a norm from $F_{v}(\sqrt{b})$ and -1 otherwise, takes the value 1 .

Let $\Sigma_{B}$ denote the set all $v \in|F|$ that do not split $B$. Class field theory implies the map $B \mapsto \Sigma_{B}$ induces a bijection between the set of isomorphism classes of quaternion algebras over $F$ and the set of finite even subsets of $|F|$; in the case $B=(a, b \mid F)$, this amounts to the product formula $\prod_{v}(a, b)_{v}=1$.

The (reduced) discriminant $\mathfrak{d}_{B}$ of $B$ is the squarefree integral ideal composed of the primes at which $B$ does not split. When $B$ is a quaternion algebra over the rational number field $F=\mathbb{Q}$, the discriminant $\mathfrak{d}_{B}$ is an integral ideal in $\mathbb{Z}$ generated by a unique positive integer $d_{B}$, which we shall also call the discriminant of $B$.

Orders. An order $R$ in $B$ is a unital subring of $B$ that is a lattice, i.e., for which $\operatorname{dim}_{\mathbb{Z}} R=\operatorname{dim}_{\mathbb{Q}} B$. A maximal order is an order that is maximal in $B$ with respect to inclusion. For example, if $\mathfrak{o}$ is an order in $F(\sqrt{a})=F \oplus F i, i^{2}=a$, and $b \in F \cap \mathfrak{o}$, then $\mathfrak{o} \oplus \mathfrak{o} j$ is an order in $(a, b \mid F)$; if $\mathfrak{o}$ is an order in $F$, then $M_{2}(\mathfrak{o})$ is an order in $M_{2}(F)$; if $\mathfrak{o}$ is maximal in $F$, then $M_{2}(\mathfrak{o})$ is maximal in $M_{2}(F)$.

An Eichler order $R \subset B$ is an intersection of two maximal orders in $B$. Let $\mathfrak{o}$ be the maximal order in the number field $F$, and $\mathfrak{o}_{v}$ its closure in $F_{v}$ for each finite place $v$ of $F$. The level of an Eichler order $R \subset B$ is the integral ideal $\mathcal{N}$ of $F$, coprime to $\mathfrak{d}_{B}$, such that for each finite place $v$ of $F$ at which $B$ splits, there exists an isomorphism $B \otimes_{F} F_{v} \rightarrow M_{2}\left(F_{v}\right)$ taking $R \otimes_{\mathfrak{o}} \mathfrak{o}_{v}$ to $\left(\begin{array}{cc}\mathfrak{o}_{v} & \mathfrak{o}_{v} \\ \mathfrak{N}_{\mathfrak{o}_{v}} & \mathfrak{o}_{v}\end{array}\right)$. When $F=\mathbb{Q}$ and $\mathcal{N}$ is generated by the positive integer $N$, we shall refer to $N$ as the level of $R$.

Indefinite rational quaternion algebras. An indefinite rational quaternion algebra is a quaternion algebra over $\mathbb{Q}$ that splits at the unique real place $\infty$ of $\mathbb{Q}$, or equivalently, for which there exists an embedding $B \hookrightarrow M_{2}(\mathbb{R})$ of $\mathbb{Q}$-algebras. Class field theory implies that the map $B \mapsto d_{B}$ induces a bijection between the set of isomorphism classes of indefinite rational quaternion algebras and the set of positive squarefree integers having an even number of prime factors.

Let $B$ be an indefinite rational quaternion algebra. The map sending an Eichler order $R \subset B$ to its level $N \in \mathbb{N}$ induces a bijection between the $B^{\times}$-conjugacy classes of Eichler orders in $B$ and the positive integers coprime to $d_{B}$. For example, if $B=M_{2}(\mathbb{Q})$, then $B$ is an indefinite rational quaternion algebra of discriminant $d_{B}=1$, and every Eichler order of (positive integral) level $N$ in $B$ is conjugate to $\left(\begin{array}{cc}\mathbb{Z} & \mathbb{Z} \\ N \mathbb{Z} & \mathbb{Z}\end{array}\right)$.

\footnotetext{
${ }^{4}$ For each embedding $B \hookrightarrow M_{2}(E)$, the reduced norm on $B$ is the pullback of the determinant on $M_{2}(E)$. Thus the notation "det", while mildly nonstandard, should introduce no confusion.
} 
Definition 2.1. For each squarefree positive integer $D$ having an even number of prime factors and each positive integer $N$ coprime to $D$, we choose a subgroup

$$
\Gamma_{0}^{D}(N)<\mathrm{SL}_{2}(\mathbb{R})
$$

as follows.

- If $D=1$, we take $\Gamma_{0}^{D}(N)=\Gamma_{0}(N)=\left(\begin{array}{cc}\mathbb{Z} & \mathbb{Z} \\ N \mathbb{Z}\end{array}\right) \cap \mathrm{SL}_{2}(\mathbb{Z})=\left(\begin{array}{c}\mathbb{Z} \\ N \mathbb{Z}\end{array}\right) \cap \mathrm{SL}_{2}(\mathbb{R})$.

- If $D \neq 1$, we choose an indefinite rational quaternion algebra $B$ of discriminant $D$, a real embedding $B \hookrightarrow M_{2}(\mathbb{R})$, and an Eichler order $R \subset B$ of level $N$. We then let $\Gamma_{0}^{D}(N)=R \cap \mathrm{SL}_{2}(\mathbb{R})$ be the image of the group of norm one units in $R$.

The group $\Gamma_{0}^{D}(N)$ is well defined in the sense that its $\mathrm{SL}_{2}(\mathbb{R})$-conjugacy class is uniquely determined by $D$ and $N$. The quotient $\Gamma_{0}^{D}(N) \backslash \mathrm{SL}_{2}(\mathbb{R})$ is compact if and only if $D \neq 1$.

2.3. Newforms on Shimura curves. We recall the notion of a (holomorphic) newform on a Shimura curve.

Shimura curves. Let $\mathbb{H}=\{x+i y: y>0\}$ be the upper half-plane. The group $\mathrm{GL}_{2}(\mathbb{R})^{+}$of real $2 \times 2$ matrices with positive determinant acts on $\mathbb{H}$ by fractional linear transformations: $\left(\begin{array}{ll}a & b \\ c & d\end{array}\right) z:=(a z+b) /(c z+d)$.

Let $\Gamma<\mathrm{SL}_{2}(\mathbb{R})$ be a lattice, that is to say a discrete subgroup of finite Haarcovolume. For example, $\Gamma_{0}^{D}(N)$ is a lattice. More generally, one attaches a lattice $\Gamma$ to each sequence of inclusions

$$
R \subset B \hookrightarrow M_{2}(\mathbb{R}),
$$

where $B$ is a quaternion algebra with one split real place over a totally real number field, $B \hookrightarrow M_{2}(\mathbb{R})$ is a fixed real embedding (unique up to conjugation), and $R \subset B$ is an order, by taking $\Gamma=R \cap \mathrm{SL}_{2}(\mathbb{R})$ to be the image of the group of norm one units in $R$.

For each lattice $\Gamma$, one defines a compact Riemann surface $X_{\Gamma}$ by suitably compactifying the quotient $\Gamma \backslash \mathbb{H}$ (see [31, §1.5]). By a Shimura curve 5 we mean an $X_{\Gamma}$ for some $\Gamma$ arising from a sequence (6). Such curves have canonical models over number fields (see 4.1 and $[31, \S 9]$ ). We call $X_{\Gamma}$ a compact Shimura curve 6 if $\Gamma \backslash \mathbb{H}$ is compact.

Newforms. For each $k \in 2 \mathbb{N}$, we recall the weight $k$ slash operator: for each function $f: \mathbb{H} \rightarrow \mathbb{C}$ and each $g \in \mathrm{GL}_{2}(\mathbb{R})^{+}$, the function $\left.f\right|_{k} g$ is given by

$$
\left.f\right|_{k} g(z):=\frac{\operatorname{det}(g)^{k / 2}}{(c z+d)^{k}} f(g z) \quad \text { if } g=\left[\begin{array}{ll}
* & * \\
c & d
\end{array}\right] .
$$

By an automorphic function of weight $k$ on $\Gamma$, we mean a smooth function $f: \mathbb{H} \rightarrow \mathbb{C}$ that satisfies $\left.f\right|_{k} \gamma=f$ for all $\gamma \in \Gamma$. The space $S_{k}(\Gamma)$ of cusp forms of weight $k$ on $\Gamma$ consists of those automorphic functions of weight $k$ that are holomorphic and vanish at the cusps of $\Gamma$, if any. One knows that $S_{k}(\Gamma)$ is finite-dimensional, and that $S_{2}(\Gamma)$ is isomorphic to the space of holomorphic 1-forms on $X_{\Gamma}$.

Let $\widetilde{\Gamma}$ denote the set of all $\alpha \in \mathrm{GL}_{2}(\mathbb{R})^{+}$for which $\Gamma \alpha \Gamma$ is a finite union of either left or right $\Gamma$-cosets. The Hecke algebra $\mathcal{H}(\Gamma)=\mathbb{C}[\Gamma \backslash \widetilde{\Gamma} / \Gamma]$, which consists of formal finite $\mathbb{C}$-linear combinations of double cosets $\Gamma \alpha \Gamma(\alpha \in \widetilde{\Gamma})$, has a natural

\footnotetext{
${ }^{5}$ This definition is not intended to be exhaustive.

${ }^{6}$ This terminology is a mild misnomer, because $X_{\Gamma}$ is in all cases a compact Riemann surface.
} 
right action on $S_{k}(\Gamma)$ that linearly extends $f\left|\Gamma \alpha \Gamma=\sum f\right|_{k} \alpha_{j}$ if $\Gamma \alpha \Gamma=\bigsqcup \Gamma \alpha_{j}$. By a newform in $S_{k}(\Gamma)$, we mean an eigenfunction of $\mathcal{H}(\Gamma) \square$ Let $\mathcal{F}_{k}(\Gamma)$ denote the set of newforms in $S_{k}(\Gamma)$. The set $\mathcal{F}_{k}(\Gamma)$ is preserved under scaling by $\mathbb{C}^{\times}$.

Multiplicity one. For each newform $f \in \mathcal{F}_{k}(\Gamma)$, there is a character $\lambda_{f}: \mathcal{H}(\Gamma) \rightarrow \mathbb{C}^{\times}$ of the Hecke algebra with the property $f \mid \varphi=\lambda_{f}(\varphi) f$ for all $\varphi \in \mathcal{H}(\Gamma)$. For general lattices $\Gamma$, a newform $f$ need not be determined by $\lambda_{f}$; in other words, the onedimensional irreducible constituents of the $\mathcal{H}(\Gamma)$-module $S_{k}(\Gamma)$ need not occur with multiplicity one. One perspective is that $\widetilde{\Gamma}$ is generally too small.

For arithmetic lattices $\Gamma$ arising from Eichler orders in quaternion algebras, one knows that $\widetilde{\Gamma}=B^{\times} \cap \mathrm{GL}_{2}(\mathbb{R})^{+}$is large, and (by a theorem of Jacquet-Langlands) that $\mathcal{H}(\Gamma)$ acts on $S_{k}(\Gamma)$ with multiplicity one.

In particular, each newform $f$ on $\Gamma=\Gamma_{0}^{D}(N)$ is determined by its character $\lambda_{f}$, the set $\mathcal{F}_{k}(\Gamma) / \mathbb{C}^{\times}$of scaling classes of newforms of given weight is finite, and the problem of recovering the values of a newform $f$ from its Hecke data $\lambda_{f}$ is meaningful.

2.4. Jacquet-Langlands correspondence. Let $B$ be an indefinite rational quaternion algebra, and let $N$ be a positive integer coprime to $d_{B}$. Set $\Gamma^{\prime}=\Gamma_{0}^{d_{B}}(N)$ and $\Gamma=\Gamma_{0}\left(d_{B} N\right)$. The Jacquet-Langlands correspondence asserts (among other things) that there is a natural bijection of finite sets

$$
\mathcal{F}_{k}\left(\Gamma^{\prime}\right) / \mathbb{C}^{\times}=\mathcal{F}_{k}(\Gamma) / \mathbb{C}^{\times}
$$

between the scaling classes of newforms on $\Gamma^{\prime}$ and those on $\Gamma$, characterized by a certain compatibility between the actions of $\mathcal{H}\left(\Gamma^{\prime}\right)$ and $\mathcal{H}(\Gamma)$. This map lifts noncanonically to a bijection $\mathcal{F}_{k}\left(\Gamma^{\prime}\right) \leftrightarrow \mathcal{F}_{k}(\Gamma)$.

Briefly, $f_{B} \in \mathcal{F}_{k}\left(\Gamma^{\prime}\right)$ corresponds to $f \in \mathcal{F}_{k}(\Gamma)$ if and only if for each prime $p$ not dividing $d_{B} N$, one has $\lambda_{f_{B}}\left(\Gamma^{\prime} \alpha^{\prime} \Gamma^{\prime}\right)=\lambda_{f}(\Gamma \alpha \Gamma)$ for some $\alpha^{\prime}$ (resp. $\alpha$ ) of integral trace and determinant $p$ in $\widetilde{\Gamma}^{\prime}$ (resp. $\widetilde{\Gamma}$ ).

Definition 2.2 (Compatibly normalized Jacquet-Langlands correspondents). Let $f_{B} \in \mathcal{F}_{k}\left(\Gamma^{\prime}\right)$ and $f \in \mathcal{F}_{k}(\Gamma)$ be Jacquet-Langlands correspondents. Write

$$
f(z)=\sum_{n \in \mathbb{N}} a_{f}(n) e(n z) .
$$

Call $f$ and $f_{B}$ compatibly normalized if $a_{f}(1)=1$ and the Petersson norms of $f$ and $f_{B}$ with respect to the standard hyperbolic measures on $\Gamma \backslash \mathbb{H}$ and $\Gamma^{\prime} \backslash \mathbb{H}$ coincide, i.e., $\int_{\Gamma \backslash \mathbb{H}} y^{k}|f(z)|^{2} \frac{d x d y}{y^{2}}=\int_{\Gamma^{\prime} \backslash \mathbb{H}} y^{k}\left|f_{B}(z)\right|^{2} \frac{d x d y}{y^{2}}$.

Remark 2.3. It is known (see [29, eq. 22] or [34]) that $\operatorname{vol}(\Gamma \backslash \mathbb{H})=\frac{\pi}{3} d_{B} N \prod_{p \mid d_{B} N}$ $\left(1+p^{-1}\right)$ and $\operatorname{vol}\left(\Gamma^{\prime} \backslash \mathbb{H}\right)=\frac{\pi}{3} d_{B} N \prod_{p \mid d_{B}}\left(1-p^{-1}\right) \prod_{p \mid N}\left(1+p^{-1}\right)$.

Remark 2.4. Let $f$ be as above, with $a_{f}(1)=1$. The coefficients $a_{f}(n)$ are determined, in an explicit and straightforward manner, by $\lambda_{f}$, hence by $\lambda_{f_{B}}$. They are algebraic integers that may be computed in a variety of software packages (such as SAGE [33]).

\footnotetext{
${ }^{7}$ The concise definition given here, which is more closely aligned with the local theory of newforms after Casselman, agrees with the classical one when $\Gamma=\Gamma_{0}(N)$.
} 
Remark 2.5. By compatibly normalizing $f$ and $f_{B}$, we have determined the individual values $f_{B}(z)$ only up to a scalar of magnitude one. However, for each pair of points $z_{1}, z_{2} \in \mathbb{H}$, the quantity

$$
\overline{f_{B}\left(z_{1}\right)} f_{B}\left(z_{2}\right)
$$

is well-defined in the sense that it is determined by $f$ (hence by $\lambda_{f}$, hence by $\lambda_{f_{B}}$ ) and by the data $R \subset B \hookrightarrow M_{2}(\mathbb{R})$ giving rise to the definition of $\Gamma^{\prime}$. Conversely, knowledge of the quantities (8) for all $z_{1}, z_{2} \in \mathbb{H}$ determines the form $f_{B}$ up to a scalar of magnitude one.

\section{EXPLICIT FORMULA}

In this section we state formulas for the values (see 33.1 ) and derivatives (see 3.4) of newforms on Shimura curves attached to squarefree level Eichler orders in indefinite rational quaternion algebras. $\$ \$ 3.2$ and 3.3 introduce notation that may be referred to as necessary.

3.1. Main formula for the values. Let $B$ be an indefinite rational quaternion algebra, let $N$ be a positive integer prime to $d_{B}$, and let $R \subset B \hookrightarrow M_{2}(\mathbb{R})$ be a sequence of inclusions giving rise to a lattice $\Gamma^{\prime}=\Gamma_{0}^{d_{B}}(N)$ as in $\$ \$ 2.2$ and 2.3 , Let $\Gamma=\Gamma_{0}\left(d_{B} N\right)$. Let $k$ be a positive even integer, and let $f_{B} \in \mathcal{F}_{k}\left(\Gamma^{\prime}\right)$ and $f \in \mathcal{F}_{k}(\Gamma)$ be compatibly normalized Jacquet-Langlands correspondents, as in Definition 2.2 of 82.4. Let $a_{f}(n)$ be the $n$th Fourier coefficient of $f$, as in (7). Our immediate goal is to give an explicit formula for the well-defined quantities $\overline{f_{B}\left(z_{1}\right)} f_{B}\left(z_{2}\right)$ (see Remark 2.5) in the special case that $N$ is squarefree.

Thus, suppose also that $N$ is squarefree. To state our formula, we must introduce some notation. Let $d$ be a divisor of $d_{B} N$. A basic consequence of Atkin-Lehner theory (see [1]) is that the Fourier coefficient $a_{f}(d)$ does not vanish (in fact, that $\left.a_{f}(d)= \pm d^{k / 2-1}\right)$, hence it makes sense to set

$$
c_{f}(d):=\frac{\mu((d, N))}{d \cdot a_{f}(d)} \quad\left(=d \frac{\mu\left(\left(d, d_{B}\right)\right)}{d} \frac{\mu(d)}{d \cdot a_{f}(d)}\right) .
$$

Here $\mu$ is the Möbius function, while $(a, b)$ denotes the greatest common divisor of a pair of integers $a$ and $b$. For each point $z=x+i y \in \mathbb{H}$ in the upper half-plane, define the matrix

$$
\sigma_{z}:=\left[\begin{array}{cc}
y^{1 / 2} & x y^{-1 / 2} \\
0 & y^{-1 / 2}
\end{array}\right]=\left[\begin{array}{ll}
y^{-1 / 2} & \\
& y^{-1 / 2}
\end{array}\right]\left[\begin{array}{cc}
1 & x \\
& 1
\end{array}\right]\left[\begin{array}{ll}
y & \\
& 1
\end{array}\right] \in \mathrm{SL}_{2}(\mathbb{R}),
$$

which has the property that it sends $i$ to $z$ under the usual action by fractional linear transformations.

We postpone the introduction of some additional, more involved notation $V_{k}$ and $R^{(d)}$ until $\S \S 3.2$ and 3.3 . Roughly, $V_{k}(L, t)$ is a rapidly-convergent weighted sum over a lattice $L<M_{2}(\mathbb{R})$, while $R^{(d)}$ is a lattice in $B \hookrightarrow M_{2}(\mathbb{R})$ that is locally dual to $R$ at primes dividing $d$. Taking that notation for granted, our main formula reads:

Theorem 3.1. For each $z_{1}=x_{1}+i y_{1}$ and $z_{2}=x_{2}+i y_{2}$ in $\mathbb{H}$, we have

$$
\left(y_{1} y_{2}\right)^{k / 2} \overline{f_{B}\left(z_{1}\right)} f_{B}\left(z_{2}\right)=\sum_{n \in \mathbb{N}} a_{f}(n) \sum_{d \mid d_{B} N} c_{f}(d) V_{k}\left(\sigma_{z_{1}}^{-1} R^{(d)} \sigma_{z_{2}} ; n / d\right) .
$$

Proof. See Appendix A.2 
Remark 3.2. Theorem 3.1 normalizes $f_{B}$ to have known Petersson norm (i.e., equal to that of $f$ ). This feature will be convenient in some expected applications. The Petersson norm of $f$ may be computed in a variety of ways (see e.g. Example 5.7).

3.2. Weighted sums over lattices. In this subsection we define $V_{k}(L, t)$, which appeared in the statement of Theorem 3.1. Let $k$ be a positive even integer, and let $L$ be a lattice in $M_{2}(\mathbb{R})$. For example, $M_{2}(\mathbb{Z})$ is a lattice in $M_{2}(\mathbb{R})$. For each positive real $x$, define the infinite sum of Bessel functions

$$
W_{k}(x):=2^{1-k} \sum_{n \in \mathbb{N}} n^{k}\left(n x K_{k-1}(n x)-K_{k}(n x)\right) .
$$

We refer the reader who is interested in how $W_{k}$ arises in nature to Appendix A.2, and especially (25).

There is a unique $\mathbb{R}$-linear map $\iota: \mathbb{C} \rightarrow M_{2}(\mathbb{R})$ sending $i$ to $\left(\begin{array}{cc}0 & 1 \\ -1 & 0\end{array}\right)$. Let $\varepsilon=$ $\left({ }^{1}{ }_{-1}\right)$. Then $\varepsilon^{2}=1$ and $\varepsilon \iota(z) \varepsilon=\iota(\bar{z})$ for all $z \in \mathbb{C}$. We have an orthogonal (with respect to the trace pairing) decomposition $M_{2}(\mathbb{R})=\iota(\mathbb{C}) \oplus \varepsilon \iota(\mathbb{C})$, which gives rise to maps $X, Y: M_{2}(\mathbb{R}) \rightarrow \mathbb{C}$ by requiring that $\alpha=\iota(X(\alpha))+\varepsilon \iota(Y(\alpha))$ for all $\alpha \in M_{2}(\mathbb{R})$. As functions on $M_{2}(\mathbb{R})$, we have det $=|X|^{2}-|Y|^{2}$. Set $P:=|X|^{2}+|Y|^{2}$. Explicitly,

$$
X(\alpha)=\frac{a+d+i(b-c)}{2}, Y(\alpha)=\frac{a-d+i(b+c)}{2}, P(\alpha)=\frac{a^{2}+b^{2}+c^{2}+d^{2}}{2}
$$

for $\alpha=\left(\begin{array}{ll}a & b \\ c & d\end{array}\right) \in M_{2}(\mathbb{R})$.

Finally, for each positive real $t$, set

$$
V_{k}(L, t):=\sum_{\substack{\alpha \in L \\ \operatorname{det}(\alpha)=t}} e^{i k \arg X(\alpha)} W_{k}(4 \pi|X(\alpha)|) .
$$

Here $e^{i k \arg X(\alpha)}=(X(\alpha) /|X(\alpha)|)^{k}$.

Remark 3.3. To see that the sum defining $V_{k}(L, t)$ converges rapidly, note that $W_{k}$ is bounded and decays exponentially, and that for each $x \geq 1$, there are at most $O_{L}\left(x^{4}\right)$ elements $\alpha \in L$ for which $\operatorname{det}(\alpha)>0$ and $|X(\alpha)| \leq x$. The latter claim follows from the inequality $2|X(\alpha)|^{2} \geq 2|X(\alpha)|^{2}-\operatorname{det}(\alpha)=P(\alpha)$.

When computing (10) numerically, one restricts the sum over $\alpha$ to those elements of $L$ for which the positive definite quadratic form $P$ takes values smaller than some fixed large cutoff. We note that this computation can be carried out effectively. Indeed, by applying an effective estimate for the $K$-Bessel functions together with the remarks in the preceeding paragraph, one reduces to bounding effectively the number of vectors $\alpha \in L$ for which $P(\alpha)$ satisfies some upper bound. This equals the number of integer points contained in a solid ellipsoid $O$ in $\mathbb{R}^{4}$, which may be bounded linearly in $\operatorname{vol}(O)$ and $\operatorname{vol}(\partial O)$ by a packing argument.

Example 3.4. If $L=M_{2}(\mathbb{Z})$, then for each positive integer $n$ we have

$$
V_{k}(L, n)=\sum_{\substack{a, b, c, d \in \mathbb{Z} \\ a d-b c=n}} e^{i k \theta} W_{k}(2 \pi r), \quad \text { where } r e^{i \theta}=a+d+i(b-c) \in \mathbb{C}^{\times} .
$$

Remark 3.5. Unlike in the case of classical (parabolic) Fourier expansions, the special functions $W_{k}$ that arise in the definition (10), and hence in Theorem 3.1] are not representation-theoretically significant. Indeed, they are somewhat arbitrary; other choices are possible, although ours is convenient owing to its direct expression 
in terms of the classical $K$-Bessel function (see $\$ 5.3$ and Appendix A.2). They are instead analogous to the test functions that arise in the approximate functional equation method for computing values of $L$-functions outside their domain of absolute convergence (see [22, §5.2]). In fact, there is a nonempty overlap between that method and our own (see Example 5.7), although neither is a special case of the other.

A generalization relevant for computing Shimura-Maass derivatives. The remainder of this subsection will be needed only in the statement of Theorem 3.9. Define derivations $\delta_{1}, \delta_{2}$ on the formal power series ring $\mathbb{Z}[[x, \bar{x}, y, \bar{y}]]$ via the table

\begin{tabular}{|l|l|l|l|l|}
\hline$j$ & $\delta_{j} x$ & $\delta_{j} \bar{x}$ & $\delta_{j} y$ & $\delta_{j} \bar{y}$ \\
\hline \hline 1 & 0 & $-\bar{y}$ & $-x$ & 0 \\
\hline 2 & 0 & $y$ & 0 & $x$ \\
\hline
\end{tabular}

For nonnegative integers $j_{1}$ and $j_{2}$, define a polynomial $p_{j_{1}, j_{2}}$ via

$$
p_{j_{1}, j_{2}}=x^{-j_{1}+j_{2}} e^{(x \bar{x}+y \bar{y}) / 2} \delta_{1}^{j_{1}} \delta_{2}^{j_{2}} e^{-(x \bar{x}+y \bar{y}) / 2} .
$$

The definition should be regarded as analogous to that of the Hermite polynomials $H_{n}(x)=(-1)^{n} e^{x^{2}} \frac{d^{n}}{d x^{n}} e^{-x^{2}}$. An induction shows that $p_{j_{1}, j_{2}}$ has integral coefficients and is independent of $x$ and $\bar{x}$, so that we may write

$$
p_{j_{1}, j_{2}}=\sum_{e_{1}, e_{2} \in \mathbb{Z}_{\geq 0}} C_{e_{1}, e_{2}}^{j_{1}, j_{2}} y^{e_{1}} \bar{y}^{e_{2}}
$$

for some integers $C_{e_{1}, e_{2}}^{j_{1}, j_{2}}$. Now set

$$
V_{k}^{j_{1}, j_{2}}(L, t):=\sum_{e_{1}, e_{2} \in \mathbb{Z}_{\geq 0}}(4 \pi)^{\left(j_{1}+j_{2}+e_{1}+e_{2}\right) / 2} C_{e_{1}, e_{2}}^{j_{1}, j_{2}} V_{k ; e_{1}, e_{2}}^{j_{1}, j_{2}}(L, t),
$$

where

$$
V_{k ; e_{1}, e_{2}}^{j_{1}, j_{2}}(L, t):=\sum_{\substack{\alpha \in L \\ \operatorname{det}(\alpha)=t}} e^{i\left(k+j_{1}+j_{2}\right) \arg X(\alpha)} Y(\alpha)^{e_{1}} \bar{Y}(\alpha)^{e_{2}} W_{k+\frac{j_{1}+j_{2}+e_{1}+e_{2}}{2}}(4 \pi|X(\alpha)|) .
$$

Remark 3.6. One can express $p_{j_{1}, j_{2}}$ in terms of Laguerre polynomials

$$
L_{\ell}^{(\alpha)}(x)=\sum_{i=0}^{\ell} \frac{(-x)^{i}}{i !}\left(\begin{array}{c}
\ell+\alpha \\
\ell-i
\end{array}\right)
$$

as $p_{j_{1}, j_{2}}(y, \bar{y})=j !(-y)^{j_{2}-j} \bar{y}^{j_{1}-j} L_{j}^{(J-j)}(y \bar{y})$ with $j=\min \left(j_{1}, j_{2}\right)$ and $J=\max \left(j_{1}, j_{2}\right)$. This identity is not essential for our purposes, so we omit the proof.

Example 3.7. $p_{0,0}=1$, hence $V_{k}^{0,0}(L, t)=V_{k}(L, t) . \quad p_{0, j_{2}}=(-y)^{j_{2}} \cdot p_{j_{1}, 0}=$ $\bar{y}^{j_{1}} \cdot p_{1, j_{2}}=(-1)^{j_{2}} y^{j_{2}-1}\left(y \bar{y}-j_{2}\right) \cdot p_{j_{1}, 1}=-\bar{y}^{j_{1}-1}\left(y \bar{y}-j_{1}\right) \cdot p_{j, j}=j ! L_{j}^{(0)}(y \bar{y})=$ $\left.e^{t} \frac{d^{j}}{d t^{j}}\left(t^{j} e^{-t}\right)\right|_{t=y \bar{y}}$.

3.3. Locally dual lattices. Let $B$ be an indefinite rational quaternion algebra, and let $R \subset B$ be an Eichler order of level $N$. We define here for each divisor $d$ of $d_{B} N$ the auxiliary lattice $R^{(d)}$ attached to $R$ that appeared in the statement of Theorem 3.1 in 3.4 
Let $R^{*}$ be the lattice dual to $R$ with respect to the trace pairing. Thus, $R$ is the set of all $\alpha \in B$ for which $\langle\alpha, r\rangle \in \mathbb{Z}$ for all $r \in R$. We have $R^{*} \supset R$.

It is a general fact that lattices in vector spaces over $\mathbb{Q}$ may be characterized locally. In our context, this means more precisely, the following: Let $p$ be a prime number, and set $B_{p}=B \otimes_{\mathbb{Z}} \mathbb{Z}_{p}=B \otimes_{\mathbb{Q}} \mathbb{Q}_{p}$. For each lattice $L<B$ (such as $L=R$ or $L=R^{*}$ ), set $L_{p}=L \otimes_{\mathbb{Z}} \mathbb{Z}_{p}$. Then $L_{p}$ is lattice in $B_{p}$. If $L, L^{\prime}$ are lattices in $B$ that satisfy $L_{p}=L_{p}^{\prime}$ for all primes $p$, then $L=L^{\prime}$.

We may characterize the dual lattice $R^{*}$ as follows:

- If $p \nmid d_{B} N$, then $R_{p}=R_{p}^{*}$.

- If $p \mid d_{B}$, then $B_{p}$ is a discrete valuation ring, $R_{p}$ is the unique maximal order in $B_{p}$, and $R_{p}^{*}$ is the inverse of its unique maximal ideal.

- If $p \mid N$, there exists an isomorphism $\iota: B_{p} \rightarrow M_{2}\left(\mathbb{Q}_{p}\right)$ taking $R_{p}$ to the Eichler order $\left(\begin{array}{cc}\mathbb{Z}_{p} & \mathbb{Z}_{p} \\ N \mathbb{Z}_{p} & \mathbb{Z}_{p}\end{array}\right)$. We then have $\iota\left(R_{p}^{*}\right)=\left(\begin{array}{cc}\mathbb{Z}_{p} & N^{-1} \mathbb{Z}_{p} \\ \mathbb{Z}_{p} & \mathbb{Z}_{p}\end{array}\right)$.

For each divisor $d$ of $d_{B} N$, there is a unique intermediate lattice $R^{*} \supset R^{(d)} \supset R$ for which

$$
R_{p}^{(d)}= \begin{cases}R_{p} & \text { if } p \nmid d, \\ R_{p}^{*} & \text { if } p \mid d\end{cases}
$$

as lattices in $B_{p}$. We have $R^{(1)}=R$ and $R^{\left(d_{B} N\right)}=R^{*}$, while $R^{(d)}$ is indeed locally dual to $R$ at the prime divisors of $d$.

3.4. Extension to Shimura-Maass derivatives. A generalization of Theorem 3.1 gives a formula for arbitrary Shimura-Maass derivatives of a newform $f_{B}$. These determine the geodesic polar expansion of $f_{B}$ about an arbitrary point, which is useful for rapidly evaluating $f_{B}$ at a large number of points.

Let $k$ be a positive even integer, and $\Gamma<\mathrm{SL}_{2}(\mathbb{R})$ a lattice. Let

$$
\delta_{k}:=y^{-k / 2-1}\left(2 i y \frac{\partial}{\partial z}+\frac{k}{2}\right) y^{k / 2}=(-4 \pi) \cdot \frac{1}{2 \pi i}\left(\frac{\partial}{\partial z}+\frac{k}{2 i y}\right)
$$

be the Shimura-Maass raising operator sending (not necesssarily holomorphic) automorphic functions on $\Gamma$ of weight $k$ to those of weight $k+2$. This agrees with the action by the element $\frac{1}{2}\left(\begin{array}{cc}1 & i \\ i & -1\end{array}\right)$ of the complexified Lie algebra of $\mathrm{SL}_{2}(\mathbb{R})$ under the usual identification of automorphic functions $f$ of weight $k^{\prime}\left(k^{\prime}=k\right.$ or $\left.k^{\prime}=k+2\right)$

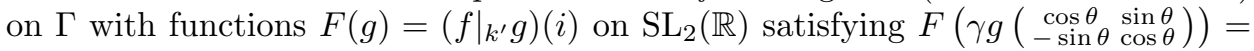
$F(g) e^{i k^{\prime} \theta}$ for all $\gamma \in \Gamma, \theta \in \mathbb{R} / 2 \pi \mathbb{Z}$. For notational simplicity, we henceforth omit the subscript $k$ from $\delta_{k}$ and write $\delta^{\ell}$ for the $\ell$-fold composition $\delta_{k+2 l-2} \circ \cdots \circ \delta_{k+2} \circ \delta_{k}$.

Example 3.8. If $\Gamma$ contains $\left(\begin{array}{cc}1 & 1 \\ 1\end{array}\right)$ and $f \in S_{k}(\Gamma)$ has the Fourier expansion $f(z)=\sum_{n \in \mathbb{N}} a_{n} q^{n}$, then its first couple of Shimura-Maass derivatives are $\delta f(z)=$ $\sum_{n \in \mathbb{N}}\left(\frac{k}{y}-4 \pi n\right) a_{n} q^{n}$ and $\delta^{2} f(z)=\sum_{n \in \mathbb{N}}\left(\frac{k(k+1)}{y^{2}}-\frac{2(k+1)(4 \pi n)}{y}+(4 \pi n)^{2}\right) a_{n} q^{n}$. For $\ell \in \mathbb{Z}_{\geq 0}$, an induction confirms that $\delta^{\ell} f$ is the weight $k+2 \ell$ automorphic function with Fourier expansion $\delta^{\ell} f(z)=y^{-\ell} \ell ! \sum_{n \in \mathbb{N}} L_{\ell}^{(k-1)}(4 \pi n y) a_{n} q^{n}$, where $L_{\ell}^{(k-1)}$ is a Laguerre polynomial as in (12). 
Theorem 3.9. With notation and assumptions as in Theorem 3.1, and $V_{k}^{j_{1}, j_{2}}$ as in (11), we have

$$
y_{1}^{\frac{k}{2}+j_{1}} y_{2}^{\frac{k}{2}+j_{2}} \overline{\delta^{j_{1}} f_{B}\left(z_{1}\right)} \delta^{j_{2}} f_{B}\left(z_{2}\right)=\sum_{n \in \mathbb{N}} a_{f}(n) \sum_{d \mid d_{B} N} c_{f}(d) V_{k}^{j_{1}, j_{2}}\left(\sigma_{z_{1}}^{-1} R^{(d)} \sigma_{z_{2}} ; \frac{n}{d}\right) .
$$

Proof. See Appendix A.2

\subsection{Complements.}

3.5.1. Specialization to CM points. The following brief remarks are not necessary for general comprehension of the paper. Let $K$ be an imaginary quadratic field. Suppose that $z=z_{1}=z_{2} \in \mathbb{H}$ is the fixed point of some embedding $K \hookrightarrow B$. Then the lattices $\sigma_{z}^{-1} R^{(d)} \sigma_{z}$ arising in Theorems 3.1 and 3.9 decompose as "sums of translates of products" of quadratic lattices tailored to the orthogonal decomposition $B=K \oplus K^{\perp}$ afforded by the trace pairing (see [28] for a more precise discussion of related issues), leading to a pleasant formula for $\overline{\delta^{j_{1}} f_{B}(z)} \delta^{j_{2}} f_{B}(z)$ involving sums of the shape

$$
\sum_{\substack{m_{1}, m_{2} \geq 0 \\ \ell_{1} m_{1}>\ell_{2} m_{2}}} a_{f}\left(\ell_{1} m_{1}-\ell_{2} m_{2}\right) r_{1}\left(m_{1}\right) r_{2}\left(m_{2}\right) W_{k+\frac{j_{1}+j_{2}+e_{1}+e_{2}}{2}}\left(4 \pi \sqrt{m_{1}}\right) .
$$

Here $\ell_{1}$ and $\ell_{2}$ are positive integers, while $r_{1}\left(m_{1}\right)$ and $r_{2}\left(m_{2}\right)$ are the Fourier coefficients of certain imaginary quadratic theta series given by

$$
r_{1}\left(m_{1}\right)=\sum_{\substack{\alpha \in \mathfrak{a}_{1} \\|\alpha|^{2}=m_{1}}}\left(\frac{\alpha}{|\alpha|}\right)^{k+j_{1}+j_{2}}, \quad r_{2}\left(m_{2}\right)=\sum_{\substack{\alpha \in \mathfrak{a}_{2} \\|\alpha|^{2}=m_{2}}} \alpha^{e_{1}} \bar{\alpha}^{e_{2}}
$$

for some (translates of) lattices $\mathfrak{a}_{1}, \mathfrak{a}_{2}$ in $K$. We do not state any precise forms of such identities here, but remark that we have implemented their derivation algorithmically. It would be interesting to compare what one gets in this way when $j_{1}=j_{2}$ with what one would get via an explicit form of Waldspurger's formula and an approximate functional equation.

In this way, one simplifies the problem of evaluating $V_{k}(L ; t)$ from one of enumerating short vectors in a rank four quadratic lattice over $\mathbb{Q}$ to one of enumerating short vectors in a pair of rank one Hermitian lattices over $K$.

3.5.2. Remarks on assumptions. We have restricted $N$ to be squarefree in our formulas only because our method of choice requires knowledge of the Fourier expansion of $f$ at each cusp of $\Gamma_{0}\left(d_{B} N\right)$ (see $\$ 5$ ). The problem of writing down such expansions for forms of nonsquarefree level, while not altogether trivial, seems orthogonal to the primary purposes of this paper (see e.g. [25, §1.9] for further discussion). For weights $k>2$, one can use the holomorphic projection method described in $\$ 5.4$ to obtain formulas valid for general levels involving the Fourier expansion of $f$ only at the cusp $\infty$. See Remark 5.8 for some musings on whether this method can be extended to the crucial case $k=2$.

3.5.3. Effectivity. As the the formulas for the values and derivatives of $f_{B}$ given in this section converge absolutely and have explicit terms, the resulting method for computing $f_{B}$ is effective (cf. Remark 3.3). We state this for emphasis as follows: 
Theorem 3.10. Let $d_{B}, N, R \subset B \hookrightarrow M_{2}(\mathbb{R}), f$, and $f_{B}$ be as in the hypotheses of Theorem 3.1. There exists an algorithm that, given $\varepsilon>0, z_{1}, z_{2} \in \mathbb{H}, j_{1}, j_{2} \in$ $\mathbb{Z}_{\geq 0}$, a basis for $R \subset B \hookrightarrow M_{2}(\mathbb{R})$, and the Fourier coefficients $a_{f}(n)$ for $n$ up to some explicit bound (depending upon $\varepsilon$ and the other parameters), computes $\overline{\delta^{j_{1}} f_{B}}\left(z_{1}\right) \delta^{j_{2}} f_{B}\left(z_{2}\right)$ to within precision $\varepsilon$.

We save an analysis of the runtime of this algorithm for a future paper. The most efficient approach that we know makes use of the ideas sketched in 3.5.1.

\section{NumericAl EXAMPLES}

In [26], we gave several examples of computations of the values and derivatives of modular forms on compact Shimura curves using a precursor of the method presented thus far. For example, we checked the rationality of their absolute values at CM points up to powers of known real periods.

Shortly after [26] was presented, we improved the method to its present form and worked out some numerical examples along the lines of the motivation discussed in 1.1. In this section, we record several such examples.

In more detail, this section is organized as follows. In 4.1, we recall how given a (modular) elliptic curve $E_{/ \mathbb{Q}}$ and an imaginary quadratic discriminant $D$ satisfying certain compatibility conditions, together with an auxiliary Atkin-Lehner operator $\tau$, one can attach a point $P(D, \tau) \in E\left(H_{D}\right)$ (with $H_{D}$ the ring class field attached to $D$ ) which is well defined up to automorphisms of $E$ and Galois conjugacy. We formulate a precise sense in which it is meaningful to compute such points (Problem 4.1). In $\$ 4.2$, we recall how such computation reduces to the evaluation of modular forms on Shimura curves. In $\$ 4.3$, we record a small sample of numerical results obtained using the formulas stated in 3 .

4.1. Setting. Let $B$ be an indefinite rational quaternion algebra, let $N$ be a positive integer coprime to $d_{B}$, and let $R \subset B \hookrightarrow M_{2}(\mathbb{R})$ be an Eichler order and fixed real embedding giving rise to $\Gamma^{\prime}=\Gamma_{0}^{d_{B}}(N)=R \cap \mathrm{SL}_{2}(\mathbb{R})$ as in $\S \S 2.2$ and 2.3, Let $X_{B}:=X_{0}^{d_{B}}(N)$ be the Shimura curve attached to $\Gamma^{\prime}$ as in 2.3 .

$C M$ points. Let $D$ be a negative quadratic discriminant, so that $D=d_{K} c^{2}$ for some $c \in \mathbb{N}$ and some imaginary quadratic field $K$ of discriminant $d_{K}<0$. There is a unique (up to isomorphism) quadratic order $\mathcal{O}=\mathcal{O}_{D}$ of discriminant $D$, constructed as $\mathcal{O}=\mathbb{Z}\left[c\left(d_{K}+\sqrt{d_{K}}\right) / 2\right]$. Let $H_{D} / K$ be the ring class extension of $K$ attached to $\mathcal{O}$, so that class field theory gives a natural isomorphism Art $: \operatorname{Pic}(\mathcal{O}) \rightarrow \operatorname{Gal}\left(H_{D} / K\right)$. Let $h(D)=\# \operatorname{Pic}(\mathcal{O})=\left[H_{D}: K\right]$ be the class number of $D$.

Call an embedding $\mathcal{O} \hookrightarrow R$ optimal if its extension to $K \hookrightarrow B$ satisfies $K \cap R=\mathcal{O}$. Let $\mathrm{CM}_{D}\left(X_{B}\right)$ denote the set of images in $X_{B}$ of fixed points in $\mathbb{H}$ for optimal embeddings $\mathcal{O} \hookrightarrow R$ (recall that $R \hookrightarrow M_{2}(\mathbb{R})$ is fixed). There is a faithful action of $\operatorname{Pic}(\mathcal{O})$ on the set of optimal embeddings $\mathcal{O} \hookrightarrow R$, which descends to a faithful action on $\mathrm{CM}_{D}\left(X_{B}\right)$ that we denote by $\zeta \cdot \mathfrak{a}$ for $\zeta \in \mathrm{CM}_{D}\left(X_{B}\right)$ and $\mathfrak{a} \in \operatorname{Pic}(\mathcal{O})$ : if $\zeta$ is fixed by an optimal embedding $j: \mathcal{O} \hookrightarrow R$, and $\gamma \in B \cap \mathrm{GL}_{2}(\mathbb{R})^{+}$is chosen to satisfy $\gamma j(\mathfrak{a}) R=R$, then $\zeta \cdot \mathfrak{a}=\gamma \zeta$.

Atkin-Lehner operators. Let $R_{+}=B \cap \mathrm{GL}_{2}(\mathbb{R})^{+}$be the monoid of positive norm elements in $R$. Call each $\tau \in R_{+}$that normalizes $\Gamma^{\prime}$ an Atkin-Lehner operator. The image in $\operatorname{Aut}\left(\Gamma^{\prime} \backslash \mathbb{H}\right)$ of the Atkin-Lehner operators is a group that we denote 
by $\operatorname{AL}\left(\Gamma^{\prime}\right)$. We have $\operatorname{AL}\left(\Gamma^{\prime}\right) \cong(\mathbb{Z} / 2)^{\omega\left(d_{B} N\right)}$, where $\omega\left(d_{B} N\right)$ is the number of prime factors of $d_{B} N$, with representatives $\tau_{d}=\prod_{p \mid d} \tau_{p}$ indexed by the positive squarefree divisors $d$ of $d_{B} N$, where:

- For $p \mid d_{B}, \tau_{p} \in R_{+}$is an arbitrary element of norm $p$.

- For $p^{\alpha} \| N, \tau_{p} \in R_{+}$is an element of norm $p^{\alpha}$ that maps to $\left(\begin{array}{cc}0 & -1 \\ p^{\alpha} & 0\end{array}\right)$ under a surjection $R \rightarrow M_{2}\left(\mathbb{Z} / p^{\beta}\right)$ taking $R$ to $\left(\begin{array}{cc}\mathbb{Z} / p^{\beta} & \mathbb{Z} / p^{\beta} \\ p^{\alpha} \mathbb{Z} / p^{\beta} & \mathbb{Z} / p^{\beta}\end{array}\right)$ for some $\beta>\alpha$.

Canonical models. We regard $X_{B}$ as an algebraic curve over $\mathbb{Q}$ with respect to its canonical model as in [31, §9], characterized as follows: for each negative quadratic discriminant $D$ and each $\zeta \in \mathrm{CM}_{D}\left(X_{B}\right)$, one has $\zeta \in X_{B}\left(H_{D}\right)$; moreover, for each $\mathfrak{a} \in \operatorname{Pic}\left(\mathcal{O}_{D}\right)$, one has $\zeta \cdot \mathfrak{a}=\zeta^{\operatorname{Art}(\mathfrak{a})}$. The group $\operatorname{AL}\left(\Gamma^{\prime}\right)$ acts on $X_{B}$ via maps defined over $\mathbb{Q}$, hence preserving $\mathrm{CM}_{D}\left(X_{B}\right)$ and commuting with $\operatorname{Gal}\left(H_{D} / K\right)$. The group $\operatorname{AL}\left(\Gamma^{\prime}\right) \times \operatorname{Gal}\left(H_{D} / K\right)$ acts transitively on $\mathrm{CM}_{D}\left(X_{B}\right)$.

Heegner points. Let $E$ be an elliptic curve over $\mathbb{Q}$ of conductor $d_{B} N$, and let $\mathcal{O}=$ $\mathcal{O}_{D} \subset K=\mathbb{Q}(\sqrt{D})$ be an imaginary quadratic order of discriminant $D$. We assume that $N$ is squarefree, that $c$ is prime to $N$, and that $\operatorname{CM}_{D}\left(X_{B}\right) \neq \emptyset$. In view of the former assumptions, the latter holds if and only if

(1) the prime divisors of $d_{B}$ do not split $K$,

(2) the prime divisors of $N$ split $K$, and

(3) $c$ is prime to $d_{B}$.

Note that these conditions determine $d_{B}$ in terms of $E$ and $K$.

The modularity theorem, the Jacquet-Langlands correspondence and Faltings's isogeny theorem imply that there is a surjective $\mathbb{Q}$-map $\varphi$ : Jac $X_{B} \rightarrow E$ arising, as in $\$ 1.2$, from some newform $f_{B} \in \mathcal{F}_{2}\left(\Gamma^{\prime}\right)$. Such maps are not unique, since post-composing one with a $\mathbb{Q}$-isogeny $E \rightarrow E$ gives another, but we may and shall pin down the $\operatorname{Aut}(E)$-orbit of $\varphi$ by requiring that it not factor through a $\mathbb{Q}$-isogeny $E \rightarrow E$ of degree $>1$.

To a $\mathrm{CM}$ point $\zeta \in \mathrm{CM}_{D}\left(X_{B}\right)$ and an Atkin-Lehner operator $\tau \in \mathrm{AL}\left(\Gamma^{\prime}\right)$, we attach a point $P(D, \tau) \in E\left(H_{D}\right)$ obtained as the image of $[\tau \zeta]-[\zeta] \in \operatorname{Pic}^{0}\left(X_{B}\right)\left(H_{D}\right) \cong$ $\operatorname{Jac}\left(X_{B}\right)\left(H_{D}\right)$ under $\varphi$. Although $P(D, \tau)$ depends upon $\zeta$, its orbit under $\operatorname{Aut}(E) \times$ $\operatorname{Gal}\left(H_{D} / K\right)$ depends only upon $D$ and $\tau$. To classify such orbits conveniently, we assume that $E$ is given by an affine equation $y^{2}+a_{1} x y+a_{3} y=x^{3}+a_{2} x^{2}+a_{4} x+a_{6}$ with coefficients in $\mathbb{Q}$ (so that the $\{ \pm 1\}$-orbits on $E$ are classified by $x$-coordinates), and also that $E$ admits complex multiplication by neither $\mathbb{Q}(i)$ nor $\mathbb{Q}(\sqrt{-3})$ (so that $\operatorname{Aut}(E)=\{ \pm 1\}) 8$ The Galois orbit of an algebraic number is classified by its minimal polynomial. Letting $x(P(D, \tau)) \in H_{D}$ denote the $x$-coordinate of $P(D, \tau)$, the following computational problem is then meaningful:

Problem 4.1. For $(E, D, \tau)$ as above, find the minimal polynomial of $x(P(D, \tau))$.

The novelty here is that we are able to address this problem via archimedean analysis even when $d_{B} \neq 1$ (

${ }^{8}$ The latter condition is imposed strictly for convenience (see [31 §6.1]). 
4.2. Method. Our method for addressing Problem4.1 is the natural one suggested by the content of $\$ 1.2$ and Theorem 3.1. Let the newform $f_{B} \in \mathcal{F}_{k}\left(\Gamma^{\prime}\right)$ realize $\varphi:$ Jac $X_{B} \rightarrow E$, as in 11.2 . Suppose that $f_{B}$ is compatibly normalized with its Jacquet-Langlands lift $f$ as in Definition 2.2 so that Theorem 3.1 applies. The Fourier coefficients $a_{f}(n)$ may be read off from the arithmetic of $E$ over finite fields in the usual way.

(1) We find a basis for $H_{1}\left(X_{B}, \mathbb{Z}\right)$, which amounts to finding generators for (the abelianization of) $\Gamma^{\prime}$. In the examples below, we either found such generators in the paper [24] or worked them out "by hand". In more complicated examples, we could use the algorithm [37] as implemented in MAGMA.

(2) Using Theorem 3.1, we compute to high precision some generators 9 for the lattice $\Lambda_{f_{B}}:=\left\{\int_{\gamma} f_{B}(z) d z: \gamma \in H_{1}\left(X_{B}, \mathbb{Z}\right)\right\}=\left\{\int_{z_{0}}^{\gamma z_{0}} f_{B}(z) d z: \gamma \in \Gamma^{\prime}\right\}$ $\left(z_{0} \in \mathbb{H}\right.$ fixed).

(3) Using the built-in functionality of SAGE/PARI, we choose a Weierstrass parametrization $\mathbb{C} / \Lambda_{E} \cong E(\mathbb{C})$ for $E$.

(4) We compute a minimal isogeny $\mathbb{C} / \Lambda_{f_{B}} \rightarrow \mathbb{C} / \Lambda_{E}$, or equivalently, a nonzero complex number $\mu$ for which $\mu \Lambda_{f_{B}}$ is contained in $\Lambda_{E}$ with minimal index. Our assumption $\operatorname{Aut}(E)=\{ \pm 1\}$ implies that $\mu$ is determined up to \pm 1 .

(5) For $\tau \in \operatorname{AL}\left(\Gamma^{\prime}\right)$ and $\gamma_{1}, \gamma_{2} \in \Gamma^{\prime}$, we compute $\int_{\gamma_{1} \zeta}^{\gamma_{2} \tau \zeta} f_{B}(z) d z$ via Theorem 3.1 and its image $P(\zeta, \tau)$ under $\mathbb{C} \rightarrow \mathbb{C} / \Lambda_{f_{B}} \stackrel{\mu}{\rightarrow} \mathbb{C} / \Lambda_{E} \cong E(\mathbb{C})$ via the elliptic_exponential functionality of SAGE/PARI. Note that $P(\zeta, \tau)$ is independent of $\gamma_{1}$ and $\gamma_{2}$, which we choose to minimize the length of the integration contour.

(6) Let $\zeta_{1}, \ldots, \zeta_{h(D)}$ be the $\operatorname{Gal}\left(H_{D} / K\right)$-orbit of some $\zeta \in \mathrm{CM}_{D}\left(X_{B}\right)$. We compute these as the fixed points for the $\operatorname{Pic}(\mathcal{O})$-orbit of some optimal embedding $\mathcal{O} \hookrightarrow R$. The minimal polynomial of $x(P(D, \tau))$ divides $\prod(t-$ $\left.x\left(P\left(\zeta_{i}, \tau\right)\right)\right) \in \mathbb{Q}[t]$, whose coefficients we compute precisely.

4.3. Results. We consider the following (Cremona-labeled) elliptic curves:

\begin{tabular}{|l|l|}
\hline$E$ & model \\
\hline \hline $15 A_{1}$ & $y^{2}+x y+y=x^{3}+x^{2}-10 x-10$ \\
\hline $21 A_{2}$ & $y^{2}+x y=x^{3}-49 x-136$ \\
\hline $35 A_{1}$ & $y^{2}+y=x^{3}+x^{2}+9 x+1$ \\
\hline $26 A_{1}$ & $y^{2}+x y+y=x^{3}-5 x-8$ \\
\hline $26 B_{2}$ & $y^{2}+x y+y=x^{3}-x^{2}-213 x-1257$ \\
\hline
\end{tabular}

The table below records some Heegner points that we found on these curves, all coming from uniformizations by compact Shimura curves $\left(d_{B} \neq 1\right)$. The points not labeled as torsion have infinite order.

\footnotetext{
${ }^{9}$ In practice, it suffices to compute only one generator to high precision.
} 


\begin{tabular}{|c|c|c|c|c|}
\hline$E$ & $D$ & $h$ & $\tau$ & minimal polynomial of $x(P(D, \tau))$ \\
\hline \multirow[t]{12}{*}{$15 A_{1}$} & -7 & 1 & $\tau_{3}$ & $x=-4$ \\
\hline & & & $\tau_{5}$ & $x=3 \quad(2$-torsion $)$ \\
\hline & & & $\tau_{15}$ & $x=4 / 7$ \\
\hline & $-7 \cdot 2^{2}$ & 1 & $\tau_{3}$ & $x=-1108 / 25=-5^{-2} 2^{2} 277^{1}$ \\
\hline & -40 & 2 & $\tau_{3}$ & $x=-29 / 32$ \\
\hline & & & $\tau_{5}$ & $x=3$ (2-torsion) \\
\hline & -43 & 1 & $\tau_{3}$ & $x=-1339 / 256=-2^{-8} 13^{1} 103^{1}$ \\
\hline & & & $\tau_{5}$ & $x=3 \quad(2$-torsion $)$ \\
\hline & & & $\tau_{15}$ & $x=-79 / 2107=-7^{-2} 43^{-1} 79^{1}$ \\
\hline & -52 & 2 & $\tau_{3}$ & $2^{10} 5^{2} x^{2}-67072 x+202177$ \\
\hline & -55 & 4 & $\tau_{3}$ & $11 x^{2}+2509 x-751$ \\
\hline & -67 & 1 & $\tau_{3}$ & $x=-461899 / 30976=-2^{-8} 11^{-2} 127^{1} 3637^{1}$ \\
\hline \multirow[t]{6}{*}{$21 A_{2}$} & -15 & 2 & $\tau_{7}$ & $x=-23 / 5$ \\
\hline & -39 & 4 & $\tau_{7}$ & $13 x^{2}-103 x+433$ \\
\hline & -43 & 1 & $\tau_{7}$ & $x=-1588 / 225=-3^{-2} 5^{-2} 2^{2} 397^{1}$ \\
\hline & -51 & 2 & $\tau_{7}$ & $x=-116 / 17=-17^{-1} 2^{2} 29^{1}$ \\
\hline & $-15 \cdot 2^{2}$ & 2 & $\tau_{7}$ & $x=-527 / 5=-5^{-1} 17^{1} 31^{1}$ \\
\hline & -67 & 1 & $\tau_{7}$ & $x=-17972 / 4225=-5^{-2} 13^{-2} 2^{2} 4493$ \\
\hline \multirow[t]{8}{*}{$35 A_{1}$} & -7 & 1 & $\tau_{5}$ & $x=-1 / 7$ \\
\hline & -8 & 1 & $\tau_{5}$ & $x=-49 / 8$ \\
\hline & -23 & 3 & $\tau_{5}$ & $23 x^{3}+5062 x^{2}+3951 x+41291$ \\
\hline & $-7 \cdot 2^{2}$ & 1 & $\tau_{5}$ & $x=-7401 / 7=-7^{-1} 3^{1} 2467^{1}$ \\
\hline & $-8 \cdot 2^{2}$ & 2 & $\tau_{5}$ & $x=-49 / 8$ \\
\hline & -43 & 1 & $\tau_{5}$ & $x=-489 / 688=-2^{-4} 43^{-1} 3^{1} 163^{1}$ \\
\hline & $-7 \cdot 3^{2}$ & 4 & $\tau_{5}$ & $7 x^{2}-13 x+73$ \\
\hline & -67 & 1 & $\tau_{5}$ & $x=-202489 / 4288=-2^{-6} 67^{-1} 7^{1} 28927^{1}$ \\
\hline \multirow[t]{2}{*}{$26 A_{1}$} & -59 & 3 & $\tau_{2}$ & $11^{4} 59^{1} x^{3}+2708827 x^{2}+2483001 x+1553249 x$ \\
\hline & -67 & 1 & $\tau_{2}$ & $-2020489 / 2815675=-5^{-2} 41^{-2} 67^{-1} 2020489^{1}$ \\
\hline \multirow[t]{2}{*}{$26 B_{2}$} & -24 & 1 & $\tau_{13}$ & $-359 / 12=-2^{-3} 3^{-1} 359^{1}$ \\
\hline & -52 & 2 & $\tau_{13}$ & point at infinity \\
\hline
\end{tabular}

We emphasize that although we have listed only the (minimal polynomial of the) $x$-coordinate, the $y$-coordinate may be recovered by solving a quadratic equation.

Remark 4.2. These points were evaluated numerically using a mixture of SAGE/PARI and $\mathrm{C}++/ \mathrm{GSL}$, and then recognized via continued fractions with the naked eye. We do not claim that our results are correct, but it is likely that 
they are, because in all cases we obtained a point with the expected field of definition. In principle, one could carry out such computations provably correctly by bounding the error terms in our explicit formulas and the heights of the points $P(D, \tau)$ (via Gross-Zagier/Zhang formulas, see [23, §5]).

The longest computations recorded in the table took a few seconds. The main computational bottleneck was the evaluation of the $K$-Bessel function. An optimized $K$-Bessel function routine as in [2] would be useful for producing a more efficient implementation. It may also be possible, as in some sophisticated implementations of "approximate functional equations," to replace the role of $K$-Bessel functions with more efficiently computable functions by applying Theorem 5.6 with a more carefully chosen test function $H(s)$.

Example 4.3. For $(E, D)=\left(35 A_{1},-23\right)$, resp. $\left(\left(26 A_{1},-59\right)\right)$, we have $h(D)=3$, so there are three Atkin-Lehner orbits of CM points of conductor $D$ on the Shimura curve $X_{0}^{d_{B}}(1)$ with $d_{B}=35$ (resp. 26). Letting $x_{1}, x_{2}, x_{3}$ denote the $x$-coordinates of the resulting points on $E(\mathbb{C})$, we compute numerically that $23^{1} \prod\left(t-x_{i}\right)$ (resp. $\left.11^{4} 59^{1} \prod\left(t-x_{i}\right)\right)$ is approximately

$$
23 t^{3}+5061.9999982 \ldots t^{2}+3950.9999999 \ldots t+41290.9999609 \ldots,
$$

respectively,

$$
11^{4} 59^{1} t^{3}+2708826.9996 \ldots t^{2}+2483000.9994 \ldots t+1553248.99991 \ldots .
$$

In each case we obtain approximately the integral-coefficient polynomial given above, whose roots generate a cubic subfield of the Hilbert class field $H_{D}$ of $\mathbb{Q}(\sqrt{D})$.

Example 4.4. We have listed only one example in which our computation returned a torsion point for "nonobvious" reasons: $E=26 B_{2}, D=-52$ and $\tau=\tau_{13}$, for which the rank of the quadratic twist $E_{D}$ is 0 .

Example 4.5. For $E=35 A, D=-63=-7 \cdot 3^{2}$, we got a point defined over the ring class field $H_{-63}=\mathbb{Q}(\sqrt{-7}, \sqrt{-3})$ of the order $\mathbb{Z}[3(1+\sqrt{-7}) / 2] \subset \mathbb{Q}(\sqrt{-7})$ of conductor 3 .

\section{General method}

Throughout this section, we assume the notation and definitions of $₫ 2$.

5.1. Overview. Let $B$ be an indefinite rational quaternion algebra, let $N$ be a positive integer coprime to $d_{B}$, let $\Gamma^{\prime}=\Gamma_{0}^{d_{B}}(N)$, let $R \subset B \hookrightarrow M_{2}(\mathbb{R})$ be the Eichler order and real embedding for which $\Gamma^{\prime}=R \cap \mathrm{SL}_{2}(\mathbb{R})$ arises as (the image of) the group of norm one units in $R$, and let $\Gamma=\Gamma_{0}\left(d_{B} N\right)$. Let $k$ be a positive even integer, and let $f_{B} \in \mathcal{F}_{k}\left(\Gamma^{\prime}\right)$ and $f \in \mathcal{F}_{k}(\Gamma)$ be compatibly normalized JacquetLanglands correspondents.

One can compute the values of $f_{B}$ in the following way:

(I) Write down an explicit form of the Shimizu correspondence. This expresses $f_{B}$ in the form

$$
\left(y_{1} y_{2}\right)^{k / 2} \overline{f_{B}\left(z_{1}\right)} f_{B}\left(z_{2}\right)=\int_{\Gamma \backslash \mathbb{H}} y^{k} \overline{f(z)} \theta_{z_{1}, z_{2}}(z) \frac{d x d y}{y^{2}},
$$

where $\theta_{z_{1}, z_{2}}$ is an explicit nonholomorphic theta series depending only upon $R \subset B \hookrightarrow M_{2}(\mathbb{R})$ and $k$. Thanks to a result of Watson [39, §2.1], doing 
so amounts to an exercise in "unadelization". We define $\theta_{z_{1}, z_{2}}$ in $\$ 5.2$ and show that it satisfies (13) in Appendix B

(II) Compute the Petersson inner product on the RHS of (13). We discuss several approaches to this problem in 5.4 . Our preferred approach (Theorem [5.6), which leads to the explicit formulas of $\$ 3$, requires also the following intermediate step:

$\left(\mathrm{I} \frac{1}{2}\right)$ Write down the constant term in the Fourier expansion of $y^{k} \overline{f(z)} \theta_{z_{1}, z_{2}}(z)$ at each cusp of $\Gamma$. We carry this out in Appendix A.1 when $N$ is squarefree.

After stating a precise form of the identity (13) in $\$ 5.2$, we turn to a discussion of the general problem of computing integrals of automorphic functions (e.g., Petersson inner products). This discussion occupies the remainder of $\$ 5$ and serves to motivate Theorem [5.6, which gives a formula for rather general such integrals via roughly a "hybrid Rankin-Selberg and approximate functional equation" approach. In Appendix A.2, we apply the procedure outlined above to prove the formulas stated in $\$ 3$,

Remark 5.1. This procedure applies also to more general automorphic quotients (such as Shimura curves associated to quaternion algebras over totally real fields), but we reserve further comments in that direction for a future paper.

5.2. Definition of Shimizu theta function. Let notation be as in 55.1 Recall the functions $X$ and $P$ defined in 33.2 . For each lattice $L<M_{2}(\mathbb{R})$ and positive reals $t$ and $y$, set

$$
U_{k}(L ; t, y):=\frac{1}{2} y \sum_{\substack{\alpha \in L \\ \operatorname{det}(\alpha)=t}} X(\alpha)^{k} e^{-2 \pi y P(\alpha)} .
$$

Fix $z_{1}, z_{2} \in \mathbb{H}$, recall the notation $\sigma_{z_{i}}$ from $\$ 3.1$ and define 10

$$
\theta_{z_{1}, z_{2}}(z):=\sum_{n \in \mathbb{Z}} U_{k}\left(\sigma_{z_{1}}^{-1} R \sigma_{z_{2}} ; n, y\right) e(n x) .
$$

Theorem 5.2 ([39], Appendix [B]). We have $\left.\theta_{z_{1}, z_{2}}\right|_{k} \gamma=\theta_{z_{1}, z_{2}}$ for all $\gamma \in \Gamma$, and the identity (13) holds.

Remark 5.3. The quadratic form ( $B$, det) represents 0 (nontrivially) if and only if $B$ is split, so that $U_{k}(L ; 0, y) \neq 0$ for some lattice $L<B$ if only if $B \cong M_{2}(\mathbb{Q})$.

5.3. Problem: computing integrals of automorphic functions. Let $M$ be a positive integer and $\Gamma=\Gamma_{0}(M) 11$ There arose in $\$ 5.1$ the problem of computing the Petersson inner product of a fixed newform against an explicit theta series on $\Gamma \backslash \mathbb{H}$. This problem is a special case of a more general one to which we now turn.

Suppose that we are given a $\Gamma$-invariant function $F: \Gamma \backslash \mathbb{H} \rightarrow \mathbb{C}$. By "given", we mean that we know the Fourier expansion

$$
F(\tau z)=\sum_{n \in \mathbb{Q}} a_{F}(n, y ; \tau) e(n x)
$$

of $F$, for each $\tau \in \mathrm{SL}_{2}(\mathbb{Z})$, to some large but finite precision. The main example to keep in mind is when $F(z)=y^{k} \overline{f_{1}(z)} f_{2}(z)$ for suitably regular automorphic

\footnotetext{
${ }^{10}$ See Appendix A.1 for the Fourier expansion of $\theta_{z_{1}, z_{2}}$ at other cusps.

${ }^{11}$ The discussion that follows applies reasonably well to any lattice $\Gamma$ with cusps.
} 
functions $f_{1}$ and $f_{2}$ of the same weight $k$, at least one of which is neither holomorphic nor a Hecke eigenform. Other approaches are available when, say, both $f_{1}$ and $f_{2}$ are holomorphic.

We do not assume any regularity of $F$, but we do assume that it is absolutely integrable on $\Gamma \backslash \mathbb{H}$. We assume also that one can compute $a_{F}(n, y ; \tau)$, to a given precision, in essentially bounded time for large $y$ and in time $(1 / y)^{O(1)}$ for small $y$.

Given such an $F$, how can we compute the integral

$$
\int F:=\int_{\Gamma \backslash \mathbb{H}} F(z) \frac{d x d y}{y^{2}} ?
$$

In applications we might wish to compute many such integrals (say a million).

5.4. Some candidate approaches. As motivation, we describe here some natural approaches to the problem stated in $\$ 5.3$

Candidate approach 1: brute force. Suppose first that $M=1$, and that the Fourier expansion (14) converges rapidly on the standard fundamental domain

$$
\mathcal{F}:=\{z=x+i y \in \mathbb{H}:|x| \leq 1 / 2,|z| \geq 1\}
$$

for $\mathrm{SL}_{2}(\mathbb{Z})$. The latter condition holds in all applications we have in mind, so we henceforth refrain from stating it explicitly in this informal discussion. Then we can compute $\int F$ by truncating its Fourier expansion and integrating each term over $\mathcal{F}$.

For general $M$, we can take as a fundamental domain for $\Gamma \backslash \mathbb{H}$ the essentially disjoint union of $\tau \mathcal{F}$ over $\tau \in \mathrm{SL}_{2}(\mathbb{Z}) / \Gamma$ Then $\int F$ is the sum over $\tau$ of the integral of $F(\tau z)$ over $\mathcal{F}$, which we compute as above.

Candidate approach 2: holomorphic projection. Suppose that $k>2$, and that $F$ is a product $F(z)=y^{k} \overline{f_{1}(z)} f_{2}(z)$ where $f_{1} \in S_{k}(\Gamma)$ is a holomorphic cusp form. Suppose also there exists $\delta>0$ such that that $f_{2}(\tau z) \ll y^{-\delta}$ for all $\tau \in \mathrm{SL}_{2}(\mathbb{Z}), y \geq 113$ Let $\langle$,$\rangle denote the Petersson "inner product" on weight k$ automorphic functions on $\Gamma$, given by $\left\langle h_{1}, h_{2}\right\rangle=\int_{\Gamma \backslash \mathbb{H}} y^{k} \overline{h_{1}(z)} h_{2}(z) \frac{d x d y}{y^{2}}$ whenever the integral converges absolutely. The pairing $\langle$,$\rangle induces the structure of a finite-dimensional Hilbert space$ on $S_{k}(\Gamma)$, so there exists a unique form $h_{2} \in S_{k}(\Gamma)$, called the holomorphic projection of $f_{2}$, with the property that $\left\langle h_{1}, h_{2}\right\rangle=\left\langle h_{1}, f_{2}\right\rangle$ for all $h_{1} \in S_{k}(\Gamma)$. Suppose that the Fourier expansion of $f_{2}$ at $\infty$ reads $f_{2}(z)=\sum a_{f_{2}}(n, y) e(n x)$, with the sum taken over $n \in \mathbb{N}$. Then, following Gross-Zagier [15, §IV.5], its holomorphic projection $h_{2}$ has the expansion $h_{2}(z)=\sum a_{h_{2}}(n) e(n z)$, where

$$
a_{h_{2}}(n)=\frac{\int_{\mathbb{R}_{+}^{\times}} y^{k / 2} a_{f_{2}}(n, y) \cdot y^{k / 2} e^{-2 \pi n y} \frac{d^{\times} y}{y}}{\int_{\mathbb{R}_{+}^{\times}} y^{k / 2} e^{-2 \pi n y} \cdot y^{k / 2} e^{-2 \pi n y} \frac{d^{\times} y}{y}} .
$$

By linear algebra on the finite-dimensional space $S_{k}(\Gamma)$, we may write $h_{2}=c f_{1}+h_{2}^{\prime}$ for some complex number $c$ and some element $h_{2}$ of $S_{k}(\Gamma)$ that is $\langle$,$\rangle -orthogonal to$ $f_{1}$. The quantity $\int F=\left\langle f_{1}, f_{2}\right\rangle=\left\langle f_{1}, h_{2}\right\rangle=c\left\langle f_{1}, f_{1}\right\rangle$ may now be computed in a number of ways (see e.g. Example 5.7).

\footnotetext{
${ }^{12}$ In implementations, the sum over $\tau \in \mathrm{SL}_{2}(\mathbb{Z}) / \Gamma$ is replaced by a sum over the cusps of $\Gamma$.

${ }^{13}$ This assumption holds in the setting of $\$ 5.1$ provided that $B \neq M_{2}(\mathbb{Q})$.
} 
Candidate approach 3: equidistribution of thickened horocycles. Our eventual method of choice (Theorem 5.6 below) is natural from several perspectives in retrospect, but was originally inspired by the following technique used by Holowinsky [20] in his work on the quantum unique ergodicity conjecture. Suppose, for simplicity, that $M=1$. Let $Y$ be a real parameter tending to $\infty$. The thickened horocycle

$$
\mathcal{R}_{Y}:=\left\{x+i y \in \mathbb{H}:|x| \leq 1 / 2, y \in\left[Y^{-1}, 2 Y^{-1}\right]\right\}
$$

contains $\asymp Y$ copies of the fundamental domain $\mathcal{F}$ for $\mathrm{SL}_{2}(\mathbb{Z}) \backslash \mathbb{H}$ (see (15)), so it is reasonable to expect that

$$
\int F \approx \frac{1}{Y} \int_{z \in \mathcal{R}_{Y}} F(z) \frac{d x d y}{y^{2}}=\frac{1}{Y} \int_{y=Y^{-1}}^{2 Y^{-1}} a_{F}(0, y ; 1) \frac{d^{\times} y}{y} .
$$

Intuitively, $\int F$ should be roughly an average of the constant term $a_{F}(0, y ; 1)$ taken over $y$ sufficiently close to 0 . This is made rigorous in the following lemma implicit in [20] (although the formulation here is our own).

Lemma 5.4. Fix a smooth compactly-supported function $h \in C_{c}^{\infty}\left(\mathbb{R}_{+}^{\times}\right)$with Mellin transform $h^{\wedge}(s)=\int_{(2)} h(y) y^{-s} \frac{d s}{2 \pi i}$ normalized so that $h^{\wedge}(1)=\int_{\mathrm{SL}_{2}(\mathbb{Z}) \backslash \mathbb{H}} \frac{d x d y}{y^{2}}$. Define a norm $S$ on the space of functions $F: \mathrm{SL}_{2}(\mathbb{Z}) \backslash \mathbb{H} \rightarrow \mathbb{C}$ by $S(F)=$ $\int_{z \in \mathcal{F}} y^{1 / 2}|F(z)| \frac{d x d y}{y^{2}}$. Let $F: \mathrm{SL}_{2}(\mathbb{Z}) \backslash \mathbb{H} \rightarrow \mathbb{C}$ be a function for which $S(F)<\infty$, and let $a_{F}(0, y ; 1)$ be its zeroth Fourier coefficient at $\infty$ as in (14). Then for each $Y \geq 1$, we have

$$
\int_{\mathrm{SL}_{2}(\mathbb{Z}) \backslash \mathbb{H}} F(z) \frac{d x d y}{y^{2}}=\frac{1}{Y} \int_{y \in \mathbb{R}_{+}^{*}} h(Y y) a_{F}(0, y ; 1) \frac{d^{\times} y}{y}+O\left(\frac{S(F)}{Y^{1 / 2}}\right),
$$

where the implied constant depends only upon $h$.

5.5. Criticism of candidate approaches. The approaches described in 55.4 work 14 but suffer some drawbacks, which we now describe.

The brute force approach, which involves integrating over $\mathcal{F}$, is somewhat slow. In applications, we are not aware of closed form expressions for the integrals over $\mathcal{F}$ that arise, so we must compute each one anew. A typical such integral might look like a linear combination of terms

$$
\int_{\substack{y=0 \\ x^{2}+y^{2} \geq 1}}^{\infty} \int_{\substack{x=-1 / 2 \\ k-1}}^{1 / 2} e^{2 \pi i n b} e^{-2 \pi c y} d x d y
$$

over some integers $b$ and nonnegative reals $c$. Caching helps, but not enough to carry out efficiently certain more advanced applications, such as Heegner point computations.

Moreover, we have in mind the natural generalization of the problem under consideration to the setting of automorphic forms on $\mathrm{GL}_{2}$ over a number field, in which the integral over $\Gamma_{0}(M) \backslash \mathbb{H}$ with its explicit fundamental domain becomes replaced by an integral over a $\left(2 r_{1}+3 r_{2}\right)$-dimensional quotient $\Delta \backslash\left(\mathbb{H}^{r_{1}} \times \mathbb{H}_{3}^{r_{2}}\right)$, or perhaps several copies of such quotients; here $\mathbb{H}_{3}$ is hyperbolic upper half-space. An explicit description of a fundamental domain for $\Delta \backslash\left(\mathbb{H}^{r_{1}} \times \mathbb{H}_{3}^{r_{2}}\right)$ seems prohibitively complicated, even for the simplest nontrivial number fields. Even granting such a

\footnotetext{
${ }^{14}$ We have experimented with the first two (using SAGE) for $F$ arising as a rather general product of squarefree level newforms, their derivatives, and Shimizu theta series; see [26].
} 
description, the problem of integrating an oscillating multivariate function over such a fundamental domain (a few million times) still seems computationally infeasible.

The holomorphic projection approach works quite well when $k$ is large enough, even over number fields. It is simpler than the other methods in the presence of nontrivial level because it requires knowledge of the Fourier coefficients $a_{F}(n, y ; \tau)$ only when $\tau=1$ (i.e., at the cusp $\infty$ ). When $k$ is small, say $k=6$ or $k=4$, the computation of the numerator of (16) becomes prohibitively slow. There is a holomorphic projection formula for $k=2$ that we have not stated here (see [15. §IV.6]), but it involves a delicate limiting procedure that seems to render it computationally ineffective. For us, the case $k=2$ is important owing to its relevance for computing modular parametrizations of elliptic curves.

Lemma 5.4 is convenient for certain theoretical applications, as in 20. However, it is not well suited for numerics: One must take $Y^{1 / 2} \gg 10^{R}$ in Lemma 5.4 to obtain $R$ digits of precision past the decimal point, but it may require time (not less than) polynomial in $Y$ to compute the integral on the RHS of (17). For applications in which high precision is needed, an algorithm requiring time exponential in the number of digits of desired precision is infeasible.

5.6. Our method of choice. We begin by recalling some background on Eisenstein series. Let $\xi(s)=\Gamma_{\mathbb{R}}(s) \zeta(s)$ be the completed Riemann zeta function, where $\Gamma_{\mathbb{R}}(s)=\pi^{-s / 2} \Gamma(s / 2)$ and $\zeta(s)=\sum_{n \in \mathbb{N}} n^{-s}(\operatorname{Re}(s)>1)$. Let $\Gamma_{\infty}=\left\{ \pm\left(\begin{array}{cc}1 & n \\ 1\end{array}\right): n \in \mathbb{Z}\right\}$ be the stabilizer in $\mathrm{SL}_{2}(\mathbb{Z})$ of the cusp $\infty$, let

$$
E_{s}(z)=\sum_{\gamma \in \Gamma_{\infty} \backslash \mathrm{SL}_{2}(\mathbb{Z})} \operatorname{Im}(\gamma z)^{s} \quad(\operatorname{Re}(s)>1)
$$

be the standard real-analytic Eisenstein series for $\mathrm{SL}_{2}(\mathbb{Z})$, and let $E_{s}^{*}(z)$ $=2 \xi(2 s) E_{s}(z)$ be its completion. The functions $E_{s}$ and $E_{s}^{*}$ descend to $\mathrm{SL}_{2}(\mathbb{Z}) \backslash \mathbb{H}$. We collect some of their standard properties in the following proposition.

Proposition 5.5 (see [21]). Let $z \in \mathbb{H}$. The function $s \mapsto E_{s}(z)$, defined initially for $\operatorname{Re}(s)>1$ by an absolutely and locally uniformly convergent series, extends to a meromorphic function on the complex plane that is holomorphic in the half-plane $\operatorname{Re}(s) \geq 1 / 2$ away from a simple pole at $s=1$. The function $s \mapsto E_{s}^{*}(z)$ extends to a meromorphic function on the complex plane, holomorphic away from simple poles at $s=1$ and $s=0$, that satisfies the functional equation $E_{s}^{*}(z)=E_{1-s}^{*}(z)$. One has $\operatorname{res}_{s=1} E_{s}(z)=\left(\int_{\mathrm{SL}_{2}(\mathbb{Z}) \backslash \mathbb{H}} \frac{d x d y}{y^{2}}\right)^{-1}$ and $\operatorname{res}_{s=1} E_{s}^{*}(z)=1$. The function $E_{s}(z)$ has moderate growth in both variables in the sense that for $\operatorname{Re}(s)$ in a fixed compact subset of $[1 / 2, \infty)$ and $z=x+$ iy in the fundamental domain $\mathcal{F}$, it satisfies $E_{s}(z) \ll(1+|s|)^{O(1)} y^{\operatorname{Re}(s)}$. The function $s \mapsto E_{s}^{*}(z)$ decays rapidly in vertical strips in the sense that if $A \in \mathbb{R}, z \in \mathbb{H}$ and $\operatorname{Re}(s) \in \mathbb{R}$ are taken to lie in fixed compact sets, then $E_{s}^{*}(z) \ll(1+|s|)^{-A}|s(s-1)|^{-1}$.

As motivation, we sketch the proof of Lemma 5.4. Let $h$ and $Y$ be as in its statement, and define $E^{Y}(z)=\sum_{\gamma \in \Gamma_{\infty} \backslash \mathrm{SL}_{2}(\mathbb{Z})} Y^{-1} h(Y \cdot \operatorname{Im}(\gamma z))$. By unfolding, $\int E^{Y} F$ gives the main term on the RHS of (17). On the other hand, Mellin inversion and a contour shift show that

$E^{Y}(z)=\int_{(2)} h^{\wedge}(s) Y^{s-1} E_{s}(z) \frac{d s}{2 \pi i}=1+Y^{-1 / 2} \int_{t \in \mathbb{R}} h^{\wedge}(1 / 2+i t) Y^{i t} E_{1 / 2+i t}(z) \frac{d t}{2 \pi}$. 
Since repeated partial integration implies that $h^{\wedge}(1 / 2+i t) \ll(1+|t|)^{-A}$ for each fixed $A \in \mathbb{R}$, we deduce from Proposition 5.5 that $E^{Y}(z)=1+O\left(Y^{-1 / 2} y^{1 / 2}\right)$ for all $z \in \mathcal{F}$. Integrating against $F$ gives (17).

We refine this argument by completing the Eisenstein series and continuing past the line $\operatorname{Re}(s)=1 / 2$, noting that unlike $E_{s}$, which has infinitely many poles in the region $0<\operatorname{Re}(s)<1 / 2$, the function $E_{s}^{*}$ is holomorphic away from $s=0$ and $s=1$. Fix $\varepsilon>0$, and let $H$ be a holomorphic function on the strip $\{s \in \mathbb{C}:-\varepsilon<\operatorname{Re}(s)<$ $1+\varepsilon\}$ satisfying the conditions $H(0)=0, H(1)=1$, and $H(s) \ll(1+|s|)^{O(1)}$. For example, one can take $H(s)=s$. Then $H(s) E_{s}^{*}$ is holomorphic away from its simple pole at $s=1$ of residue 1 and decays rapidly in the strip $-\varepsilon<\operatorname{Re}(s)<1+\varepsilon$, so for each $\delta \in(0, \varepsilon)$, we have the fundamental identity

$$
\begin{aligned}
1=\operatorname{res}_{s=1} H(s) E_{s}^{*} & =\left(\int_{(1+\delta)}-\int_{(-\delta)}\right) H(s) E_{s}^{*} \frac{d s}{2 \pi i} \\
& =\int_{(1+\delta)}(H(s)-H(1-s)) E_{s}^{*} \frac{d s}{2 \pi i} .
\end{aligned}
$$

In the final step, we used the functional equation $E_{s}^{*}=E_{1-s}^{*}$.

We would like to integrate (18) against $F: \Gamma \backslash \mathbb{H} \rightarrow \mathbb{C}$ and interchange the order of integration to obtain

$$
\int F=\int_{(1+\delta)}(H(s)-H(1-s)) 2 \xi(2 s)\left(\int F E_{s}\right) \frac{d s}{2 \pi i} .
$$

By taking $\delta$ sufficiently small, we see that this interchange is justified provided that $|F(z)| \ll y^{-\alpha}$ for some fixed $\alpha>0$ and all $z=x+i y$ with $y \geq 1$. Unfolding gives $\int F E_{s}=\int_{y \in \mathbb{R}_{+}^{\times}} a_{0}(0, y ; 1) y^{s-1} d^{\times} y$.

If $\Gamma$ is a finite index subgroup of $\mathrm{SL}_{2}(\mathbb{Z})$ and $F$ is on $\Gamma \backslash \mathbb{H}$, then applying the above argument to its pushforward $z \mapsto \sum_{\Gamma \backslash \mathrm{SL}_{2}(\mathbb{Z})} F(\tau z)$ on $\mathrm{SL}_{2}(\mathbb{Z}) \backslash \mathbb{H}$ yields:

Theorem 5.6. Let $\Gamma$ be a finite index subgroup of $\mathrm{SL}_{2}(\mathbb{Z})$. Let $F: \Gamma \backslash \mathbb{H} \rightarrow \mathbb{C}$ be a bounded measurable function satisfying $F(\tau z) \ll y^{-\alpha}$ for some fixed $\alpha>0$, almost all $z=x+$ iy with $y \geq 1$, and all $\tau \in \mathrm{SL}_{2}(\mathbb{Z})$. Let $\varepsilon>0$, and let $H$ be a holomorphic function on $\{s \in \mathbb{C}:-\varepsilon<\operatorname{Re}(s)<1+\varepsilon\}$ satisfying $H(0)=0, H(1)=1$, and $H(s) \ll(1+|s|)^{O(1)}$. Then for $\delta \in(0, \min (\alpha, \varepsilon))$, we have

$\int_{\Gamma \backslash \mathbb{H}} F(z) \frac{d x d y}{y^{2}}=\int_{(1+\delta)}(H(s)-H(1-s)) 2 \xi(2 s) \sum_{\tau \in \Gamma \backslash \mathrm{SL}_{2}(\mathbb{Z})} a_{F}(0, \cdot ; \tau)^{\wedge}(1-s) \frac{d s}{2 \pi i}$.

Here $a_{F}(0, y ; \tau)$ is the constant term of the Fourier expansion of $F(\tau z)$ as in (14), while

$$
a_{F}(0, \cdot ; \tau)^{\wedge}(1-s):=\int_{y \in \mathbb{R}_{+}^{\times}} a_{F}(0, y ; \tau) y^{s-1} d^{\times} y .
$$

Example 5.7. We give an example in which Theorem 5.6 specializes to the approximate functional equation applied to Rankin-Selberg $L$-functions. Take $\Gamma=\mathrm{SL}_{2}(\mathbb{Z})$, $k=12$, and let $\Delta(z)=q \prod\left(1-q^{n}\right)^{24}=\sum a_{\Delta}(n) q^{n} \in S_{k}(\Gamma)$ be as in $\$ 1.4$. Define $F: \Gamma \backslash \mathbb{H} \rightarrow \mathbb{C}$ by the formula $F(z)=y^{k}|\Delta(z)|^{2}$. The constant term of $F$ is $a_{F}(0, y ; 1)=y^{k} \sum\left|a_{\Delta}(n)\right|^{2} e^{-4 \pi n y}$, which has the Mellin transform (for $\operatorname{Re}(s)>1$ )

$$
a_{F}(0, \cdot ; 1)^{\wedge}(1-s)=\frac{\Gamma(k-1+s)}{(4 \pi)^{k-1+s}} \sum_{n \in \mathbb{N}} \frac{\left|a_{\Delta}(n)\right|^{2}}{n^{k-1+s}}=: \frac{\Gamma(k-1+s)}{(4 \pi)^{k-1+s}} \frac{L(\Delta \times \Delta, s)}{\zeta(2 s)} .
$$


Thus Theorem 5.6 reads

$$
\begin{aligned}
\int_{\mathrm{SL}_{2}(\mathbb{Z}) \backslash \mathbb{H}} y^{k}|\Delta(z)|^{2} \frac{d x d y}{y^{2}} & =\int_{(1+\delta)}(H(s)-H(1-s)) 2 \xi(2 s) a_{F}(0, \cdot ; 1)^{\wedge}(1-s) \frac{d s}{2 \pi i} \\
& =2 \sum_{n \in \mathbb{N}} \frac{\left|a_{\Delta}(n)\right|^{2}}{(4 \pi n)^{k-1}} \sum_{d \in \mathbb{N}} \Phi_{k, H}\left(4 \pi^{2} d^{2} n\right),
\end{aligned}
$$

where $\Phi_{k, H}(y)=\int_{(1+\delta)}(H(s)-H(1-s)) \Gamma(s) \Gamma(k-1+s) y^{-s} \frac{d s}{2 \pi i}$. For the choice $H(s)=s$, one can verify that $\Phi_{k, H}(y)=y^{(k-1) / 2}\left(4 \sqrt{y} K_{k-2}(2 \sqrt{y})-2 K_{k-1}(2 \sqrt{y})\right)$ (see Lemma A.4). The result obtained is equivalent to what one would get by first expressing the Petersson norm of $\Delta$ in terms of the residue of $L(\Delta \times \Delta, s)$ at $s=1$ via the Rankin-Selberg method, and then computing that residue via an approximate functional equation. The "two proofs" are essentially rearrangements of one another.

Remark 5.8. Theorem 5.6 allows one to compute effectively the holomorphic projection of certain weight $k$ automorphic functions, even in the delicate case $k=2$. However, it requires knowledge of Fourier expansions at all cusps. It would be interesting to have an effective method for computing holomorphic projections using Fourier expansions only at the cusp $\infty$ when $k=2$. Conceivably one could redo the above argument with the nonholomorphic weight $k$ Poincaré series and its recurrence relation under $s \mapsto s+1$ taking the place of the nonholomorphic weight 0 Eisenstein series and its functional equation under $s \mapsto 1-s$, but we have not attempted to do so.

Remark 5.9. Theorem 5.6 is also of theoretical interest, because it gives a way to evaluate integrals of automorphic functions $F$ for which one cannot satisfactorily control the norm $S(F)$ of Lemma 5.4. We exploit this property in a paper under preparation to bound periods of restrictions of Hilbert modular forms.

\section{Appendix A. Proof of Formulas}

In this section we show how the identity of Theorem 5.2 and the method for computing Petersson inner products afforded by Theorem 5.6 imply the identites stated in 93 ,

A.1. Fourier expansions at various cusps. Preserve the notation of $\$ \$ 5.1$ and 5.2. Let $F: \mathbb{H} \rightarrow \mathbb{C}$ be the function $F(z)=y^{k} \overline{f(z)} \theta_{z_{1}, z_{2}}(z)$, which arose as the integrand of (13). Owing to the identity $\operatorname{Im}(\tau z)=\operatorname{Im}(z) /|c z+d|^{2}$ for $\tau=\left(\begin{array}{ll}* * \\ c & d\end{array}\right) \in$ $\mathrm{SL}_{2}(\mathbb{R})$, we have $F(\tau z)=\left.y^{k} \overline{\left.f\right|_{k} \tau(z)} \theta_{z_{1}, z_{2}}\right|_{k} \tau(z)$ for all $\tau \in \mathrm{SL}_{2}(\mathbb{R})$. In particular, $F(\gamma z)=F(z)$ for all $\gamma \in \Gamma$. For each $\tau \in \mathrm{SL}_{2}(\mathbb{Z})$, write $F(\tau z)=\sum a_{F}(n, y ; \tau) e(n x)$ for the Fourier expansion of $F(\tau z)$, where the sum is over $n$ in $(1 / w) \mathbb{Z}$ with $w$ the width of the cusp $\tau \infty$.

Suppose now that $N$, the level of the Eichler order $R$, is squarefree. Our aim in this subsection is to evaluate the sum, taken over $\tau \in \Gamma \backslash \mathrm{SL}_{2}(\mathbb{Z})$, of the "constant terms" $a_{F}(0, y ; \tau)$ of $F$. For a lattice $L<M_{2}(\mathbb{R})$ and positive reals $t$ and $y$, set

$$
\widetilde{U}_{k}(L ; t, y):=y^{k} U_{k}(L ; t, y) e^{-2 \pi t y} .
$$

Theorem A.1. With $c_{f}(d)$ as in (9), we have

$$
\sum_{\tau \in \Gamma_{0}\left(d_{B} N\right) \backslash \mathrm{SL}_{2}(\mathbb{Z})} a_{F}(0, y ; \tau)=\sum_{n \in \mathbb{N}} a_{f}(n) \sum_{d \mid d_{B} N} c_{f}(d) \widetilde{U}_{k}\left(\sigma_{z_{1}}^{-1} R^{(d)} \sigma_{z_{2}} ; n / d, y\right) .
$$


The proof is a calculation that occupies the remainder of this subsection.

Coset representatives. For each positive integer $M$, the natural map $\mathrm{SL}_{2}(\mathbb{Z}) \rightarrow$ $\mathrm{SL}_{2}(\mathbb{Z} / M)$ is surjective. By the Chinese remainder theorem, it follows that for each divisor $d$ of $d_{B} N$, there exists an element $\tau_{d} \in \mathrm{SL}_{2}(\mathbb{Z})$ such that

$$
\tau_{d} \equiv\left[\begin{array}{cc}
0 & 1 \\
-1 & 0
\end{array}\right] \quad \bmod d, \quad \tau_{d} \equiv\left[\begin{array}{ll}
1 & 0 \\
0 & 1
\end{array}\right] \quad \bmod \frac{d_{B} N}{d} .
$$

The difference between two such elements $\tau_{d}$ belongs to the normal subgroup $\Gamma\left(d_{B} N\right)=\left\{\gamma \in \mathrm{SL}_{2}(\mathbb{Z}): \gamma \equiv 1 \bmod d_{B} N\right\}$ of $\Gamma$. The width of the cusp $\tau_{d} \infty$ is $d$. For each real $x$ and nonzero real $y$, set

$$
n(x)=\left[\begin{array}{ll}
1 & x \\
0 & 1
\end{array}\right] \in \mathrm{SL}_{2}(\mathbb{R}), \quad a(y)=\left[\begin{array}{cc}
y^{1 / 2} & 0 \\
0 & y^{-1 / 2}
\end{array}\right] \in \mathrm{SL}_{2}(\mathbb{R}) .
$$

Recall that $\Gamma=\Gamma_{0}\left(d_{B} N\right)$ with $d_{B} N$ squarefree. The following lemma is well known.

Lemma A.2. Let $d$ traverse the positive divisors of $d_{B} N$, and let $j$ traverse a set of representatives for $\mathbb{Z} / d$. Then the matrices $\tau_{d} n(j)$ traverse a set of representatives for $\Gamma \backslash \mathrm{SL}_{2}(\mathbb{Z})$.

Fourier expansion of $f$. Let $d$ be a divisor of $d_{B} N$. The newform $f \in \mathcal{F}_{k}\left(d_{B} N\right)$ has squarefree level. Let $a_{f}(n)$ be the $n$th Fourier coefficient of $f$, as in (7). By a well-known result of Atkin-Lehner, it transforms under $\tau_{d} a(d)$ via $\left.f\right|_{k} \tau_{d} a(d)(z)=$ $\frac{\mu(d) d^{k / 2-1}}{a_{f}(d)} f(z)$. Since $\left.f\right|_{k} a(d)^{-1}(z)=d^{-k / 2} f(z / d)$, it follows that

$$
\left.f\right|_{k} \tau_{d}(z)=\frac{\mu(d)}{d \cdot a_{f}(d)} f(z / d)=\frac{\mu(d)}{d \cdot a_{f}(d)} \sum_{n \in \mathbb{N}} a_{f}(n) e((n / d) x) e^{-2 \pi(n / d) y} .
$$

Fourier expansion of $\theta_{z_{1}, z_{2}}$. Let $d$ be a divisor of $d_{B} N$. One can compute the Fourier expansion of $\left.\theta_{z_{1}, z_{2}}\right|_{k} \tau_{d}$ via the adelic Weil representation (see Remark B.4). The result obtained is that

$$
\left.\theta_{z_{1}, z_{2}}\right|_{k} \tau_{d}(z)=\frac{\mu\left(\left(d, d_{B}\right)\right)}{d} \sum_{n \in \mathbb{Z}} U_{k}\left(\sigma_{z_{1}}^{-1} R^{(d)} \sigma_{z_{2}} ; n / d, y\right) e((n / d) x) .
$$

Proof of Theorem A.1, Let $d$ be a divisor of $d_{B} N$. By (19), (20), and the definition (9) of $c_{f}(d)$, the constant term of $F\left(\tau_{d} z\right)$ is

$$
\begin{aligned}
a_{F}\left(0, y ; \tau_{d}\right) & =y^{k} \frac{\mu(d)}{d \cdot a_{f}(d)} \cdot \frac{\mu\left(\left(d, d_{B}\right)\right)}{d} \sum_{n \in \mathbb{N}} a_{f}(n) U_{k}\left(\sigma_{z_{1}}^{-1} R^{(d)} \sigma_{z_{2}} ; n / d, y\right) e^{-2 \pi(n / d) y} \\
& =\frac{c_{f}(d)}{d} \sum_{n \in \mathbb{N}} a_{f}(n) \widetilde{U}_{k}\left(\sigma_{z_{1}}^{-1} R^{(d)} \sigma_{z_{2}} ; n / d, y\right) .
\end{aligned}
$$

A direct calculation shows that $a_{F}\left(0, y ; \tau_{d} n(j)\right)=a_{F}\left(0, y ; \tau_{d}\right)$ for all integers $j$, so Lemma A.2 gives

$$
\begin{aligned}
\sum_{\tau \in \Gamma_{0}\left(d_{B} N\right) \backslash \mathrm{SL}_{2}(\mathbb{Z})} a_{F}(0, y ; \tau) & =\sum_{d \mid d_{B} N} \sum_{j \in \mathbb{Z} / d} a_{F}\left(0, y ; \tau_{d} n(j)\right) \\
& =\sum_{n \in \mathbb{N}} a_{f}(n) \sum_{d \mid d_{B} N} c_{f}(d) \widetilde{U}_{k}\left(\sigma_{z_{1}}^{-1} R^{(d)} \sigma_{z_{2}} ; n / d, y\right) .
\end{aligned}
$$


A.2. Proofs of formulas stated in $\S 3$. Let notation be as in $\$ 5.1$, and set $F(z)=$ $y^{k} \overline{f(z)} \theta_{z_{1}, z_{2}}(z)$. Theorem 5.2 reads

$$
\left(y_{1} y_{2}\right)^{k / 2} \overline{f_{B}\left(z_{1}\right)} f_{B}\left(z_{2}\right)=\int_{\Gamma \backslash \mathbb{H}} F(z) \frac{d x d y}{y^{2}} .
$$

We apply Theorem 5.6 with $\varepsilon=100, H(s)=s$, and $\delta=10$, giving

$$
\int_{\Gamma \backslash \mathbb{H}} F(z) \frac{d x d y}{y^{2}}=\int_{(10)}(2 s-1) 2 \xi(2 s) \sum_{\tau \in \Gamma \backslash \mathrm{SL}_{2}(\mathbb{Z})} a_{F}(0, \cdot ; \tau)^{\wedge}(1-s) \frac{d s}{2 \pi i} .
$$

Suppose now that $N$ is squarefree. By Theorem A.1, we then have

$\sum_{\tau \in \Gamma \backslash \mathrm{SL}_{2}(\mathbb{Z})} a_{F}(0, \cdot ; \tau)^{\wedge}(1-s)=\sum_{n \in \mathbb{N}} a_{f}(n) \sum_{d \mid d_{B} N} c_{f}(d) \widetilde{U}_{k}\left(\sigma_{z_{1}}^{-1} R^{(d)} \sigma_{z_{2}} ; n / d, \cdot\right)^{\wedge}(1-s)$.

Lemma A.3. For each lattice $L<M_{2}(\mathbb{R})$ and positive real $t$,

$$
\int_{(10)}(2 s-1) 2 \xi(2 s) \widetilde{U}_{k}(L ; t, \cdot)^{\wedge}(1-s) \frac{d s}{2 \pi i}=V_{k}(L, t) .
$$

Proof of Theorem 3.1, assuming Lemma A.3. Combine (21), (22), (23) and Lemma A.3.

Proof of Theorem 3.9. This is obtained from Theorem 3.1 by differentiating. We omit the details, but remark that the resulting formula has convincingly passed all numerical tests.

To prove Lemma A.3, we need the following technical lemma.

Lemma A.4. For each positive real $x$ and $\nu \in \mathbb{C}$ with $\operatorname{Re}(\nu) \geq 0$,

$$
\int_{(10)}\left(s-\frac{1}{2}\right) \frac{\Gamma(s) \Gamma(s+\nu)}{(x / 2)^{2 s+\nu}} \frac{d s}{2 \pi i}=x K_{\nu-1}(x)-K_{\nu}(x) .
$$

Proof. Mellin inversion and the integral formula $\int_{0}^{\infty} x^{s} K_{\nu}(2 x) d^{\times} x=\frac{1}{4} \Gamma\left(\frac{s+\nu}{2}\right)$ $\Gamma\left(\frac{s-\nu}{2}\right)$ valid for $\operatorname{Re}(s \pm \nu)>0$ (see [11, 6.561.16]) show that for $\operatorname{Re}(\nu)>-5$,

$$
K_{\nu}(x)=\frac{1}{4} \int_{(\operatorname{Re}(\nu)+5)} \frac{\Gamma\left(\frac{s+\nu}{2}\right) \Gamma\left(\frac{s-\nu}{2}\right)}{(x / 2)^{s}} \frac{d s}{2 \pi i}=\frac{1}{2} \int_{(10)} \frac{\Gamma(s) \Gamma(s+\nu)}{(x / 2)^{2 s+\nu}} \frac{d s}{2 \pi i} .
$$

For $\operatorname{Re}(\nu)>-4$, the identity $s \Gamma(s)=\Gamma(s+1)$ gives

$$
x K_{\nu-1}(x)=\int_{(9)} \frac{\Gamma(s) \Gamma(s+\nu-1)}{(x / 2)^{2 s+\nu-2}} \frac{d s}{2 \pi i}=\int_{(10)} s \frac{\Gamma(s) \Gamma(s+\nu)}{(x / 2)^{2 s+\nu}} \frac{d s}{2 \pi i} .
$$

Subtracting, we are done.

Proof of Lemma A.3. Since $P(\alpha)+\operatorname{det}(\alpha)=2|X(\alpha)|^{2}$, we see that

$$
\widetilde{U}_{k}(L ; t, y)=\frac{y^{k+1}}{2} \sum_{\substack{\alpha \in L \\ \operatorname{det}(\alpha)=t}} X(\alpha)^{k} e^{-2 \pi(P(\alpha)+t) y}=\frac{y^{k+1}}{2} \sum_{\substack{\alpha \in L \\ \operatorname{det}(\alpha)=t}} X(\alpha)^{k} e^{-4 \pi|X(\alpha)|^{2} y} .
$$


Thus for each $s \in \mathbb{C}$ with $\operatorname{Re}(s)=10$,

$$
\begin{aligned}
\widetilde{U}_{k}(L ; t, \cdot)^{\wedge}(1-s) & =\int_{y \in \mathbb{R}_{+}^{\times}} \widetilde{U}_{k}(L ; t, y) y^{s-1} d^{\times} y \\
& =\frac{1}{2} \sum_{\substack{\alpha \in L \\
\operatorname{det}(\alpha)=t}} X(\alpha)^{k} \frac{\Gamma(s+k)}{\left(4 \pi|X(\alpha)|^{2}\right)^{s+k}} \\
& =\sum_{\substack{\alpha \in L \\
\operatorname{det}(\alpha)=t}} e^{i k \arg X(\alpha)} Z_{k}(4 \pi|X(\alpha)| ; s),
\end{aligned}
$$

where $Z_{k}(x ; s):=2^{-k-1} \pi^{s} \Gamma(s+k)(x / 2)^{-2 s-k}$; here the interchange of summation and integration is justified by absolute convergence. Since $\xi(2 s) \pi^{s}=$ $\Gamma(s) \sum_{m \in \mathbb{N}} m^{-2 s}$, it follows from Lemma A.4 that

$$
\begin{aligned}
\int_{(10)}(2 s-1) 2 \xi(2 s) Z_{k}(x ; s) \frac{d s}{2 \pi i} & =2^{1-k} \sum_{m \in \mathbb{N}} m^{k} \int_{(10)}\left(s-\frac{1}{2}\right) \frac{\Gamma(s) \Gamma(s+k)}{(m x / 2)^{2 s+k}} \frac{d s}{2 \pi i} \\
& =2^{1-k} \sum_{m \in \mathbb{N}} m^{k}\left(m x K_{k-1}(m x)-K_{k}(m x)\right) \\
& =W_{k}(x) .
\end{aligned}
$$

Combining (24) and (25) (with $x=4 \pi|X(\alpha)|$ ), we conclude that

$$
\int_{(10)}(2 s-1) 2 \xi(2 s) \widetilde{U}_{k}(L ; t, \cdot)^{\wedge}(1-s) \frac{d s}{2 \pi i}=\sum_{\substack{\alpha \in L \\ \operatorname{det}(\alpha)=t}} e^{i k \arg X(\alpha)} W_{k}(4 \pi|X(\alpha)|),
$$

which equals $V_{k}(L, t)$ by definition.

\section{Appendix B. Explicit Shimizu correspondence}

In this appendix, we explain how Theorem 5.2 follows from the Shimizu correspondence as pinned down by Watson [39, Thm 1].

B.1. Notation, measures. Let $B$ be an indefinite rational quaternion algebra. Recall the notation from $\S \$ 2.2$ and 2.3 . The discussion that follows applies to nonsplit $B$ as well as to $B=M_{2}(\mathbb{Q})$. We regard $B, B^{\times}, P B^{\times}, B^{1}:=\left\{b \in B^{\times}\right.$: $\operatorname{det}(b)=1\}$, and $Z\left(B^{\times}\right)=$center of $B^{\times}$as algebraic groups over $\mathbb{Q}$.

Let $\mathbb{A}=\prod^{\prime} \mathbb{Q}_{v}=\mathbb{R} \times \hat{\mathbb{Q}}\left(\hat{\mathbb{Q}}=\prod_{p}^{\prime} \mathbb{Q}_{p}\right)$ be the adele ring of $\mathbb{Q}$. For $G$ an algebraic group over $\mathbb{Q}$, write $G_{v}=G\left(\mathbb{Q}_{v}\right)$. We identify $G(\mathbb{A})=G_{\infty} \times G(\hat{\mathbb{Q}})$ without mention.

Let $G_{\infty+}$ be the topologically connected component of $G_{\infty}$, which for $G=$ $B^{\times}$or $P B^{\times}$is the subgroup of positive determinant elements. We fix compatible identifications $B_{\infty}=M_{2}(\mathbb{R})$ and $B_{\infty}^{\times}=\mathrm{GL}_{2}(\mathbb{R})$, and let $K_{\infty}=\mathrm{SO}(2) \subset B_{\infty+}^{\times}$be the maximal compact connected subgroup of $B_{\infty+}^{\times}$stabilizing $i \in \mathbb{H}$.

Let $X=$ either $\mathbb{Q}_{v}$ or $B_{v}$. Define $\langle\rangle:, X \times X \rightarrow \mathbb{Q}_{v}$ by $\mathbb{Q}_{v} \times \mathbb{Q}_{v} \ni(x, y) \mapsto x y$ and $B_{v} \times B_{v} \ni(\alpha, \beta) \mapsto \operatorname{tr}\left(\alpha \beta^{\iota}\right)=\alpha \beta^{\iota}+\beta \alpha^{\iota}$. Let $\mathcal{S}(X)$ denote the space of Schwarz-Bruhat functions on $X$. Let $\mathbf{e}=\prod \mathbf{e}_{v} \in \operatorname{Hom}\left(\mathbb{A} / \mathbb{Q}, S^{1}\right)$ be the standard 
additive character, characterized by requiring $\mathbf{e}_{\infty}(x)=e(x):=e^{2 \pi i x}$. Let $d x$ be the Haar measure on $X$ for which the Fourier transform $\mathcal{F}: \mathcal{S}(X) \rightarrow \mathcal{S}(X)$ given by $\mathcal{F} \varphi(y):=\int_{B_{v}} \varphi(x) e_{v}(\langle x, y\rangle) d x$ satisfies $\mathcal{F} \mathcal{F} \varphi(x)=\varphi(-x)$. For $x \in X$, let $|x|$ be its modulus, so that $[y \mapsto x y]^{*} d y=|x| d y$. Let $d^{\times} x$ be the measure $\zeta_{v}(1)|x|^{-1} d x$ on $X^{\times}$, where $\zeta_{p}(s):=\left(1-p^{-s}\right)^{-1}$ and $\zeta_{\infty}(s):=\pi^{-s / 2} \Gamma(s / 2)$.

The measures so-defined on $\mathbb{Q}_{v}^{\times}$and $B_{v}^{\times}$induce measures $d^{\times} \alpha$ on $P B_{v}^{\times}$and $d^{(1)} \alpha$ on $B_{v}^{1}$ via the short exact sequences $1 \rightarrow B_{v}^{1} \rightarrow B_{v}^{\times} \stackrel{\nu}{\rightarrow} \mathbb{Q}_{v}^{\times} \rightarrow 1$ and $1 \rightarrow \mathbb{Q}_{v}^{\times} \rightarrow$ $B_{v}^{\times} \rightarrow P B_{v}^{\times} \rightarrow 1$. This normalization is consistent with [39, 2.1.2]. For $p \nmid d_{B}$ (resp. $\left.p \mid d_{B}\right)$, the measure $d^{(1)} \alpha$ assigns volume $\zeta_{p}(2)^{-1}\left(\operatorname{resp} .(p-1)^{-1} \zeta_{p}(2)^{-1}\right)$ to the group of norm one units in a maximal order in $B_{p}$. Let $\sigma_{z}(z \in \mathbb{H})$ be as in 3.1. Then on $B_{\infty}^{1}$, the measure $d^{(1)} \alpha$ is given by

$$
d^{(1)} \alpha=\frac{1}{2} d x \frac{d^{\times} y}{|y|} d \theta \quad \text { if } \alpha=\sigma_{x+i y}\left[\begin{array}{cc}
\cos (\theta) & \sin (\theta) \\
-\sin (\theta) & \cos (\theta)
\end{array}\right] .
$$

The product $d^{(1)} \alpha:=\prod_{v} d^{(1)} \alpha_{v}$ of measures on $B_{v}^{1}$ converges to a measure on $B^{1}(\mathbb{A})$, inducing a quotient measure on $\left[B^{1}\right]:=B^{1}(\mathbb{Q}) \backslash B^{1}(\mathbb{A})$ by giving $B^{1}(\mathbb{Q})$ the counting measure. We may define similarly a product measure $d^{\times} \alpha:=\prod_{v} d^{\times} \alpha_{v}$ on $P B^{\times}(\mathbb{A})$ inducing a quotient measure on $\left[P B^{\times}\right]:=P B^{\times}(\mathbb{Q}) \backslash P B^{\times}(\mathbb{A})$.

B.2. Adelization. Let $R \subset B \hookrightarrow M_{2}(\mathbb{R})$ be an Eichler order and real embedding giving rise to the lattice $\Gamma=R \cap \mathrm{SL}_{2}(\mathbb{R})<\mathrm{SL}_{2}(\mathbb{R})$. Let $R_{p}=R \otimes_{\mathbb{Z}} \mathbb{Z}_{p}$ and $\hat{R}=\prod R_{p} \subset B(\hat{\mathbb{Q}})$. If $\mathcal{F}(\Gamma \backslash \mathbb{H})$ is a fundamental domain for $\Gamma \backslash \mathbb{H}$, then

$$
\left\{\sigma_{z}\left(\begin{array}{cc}
\cos \theta & \sin \theta \\
-\sin \theta & \cos \theta
\end{array}\right) \times \kappa_{0}: z \in \mathcal{F}(\Gamma \backslash \mathbb{H}), 0 \leq \theta<\pi, \kappa_{0} \in \hat{R}^{\times}\right\}
$$

is a fundamental domain for $\left[\mathrm{SL}_{2}\right]$. Fix a positive even integer $k$. The following lemma is a consequence of the strong approximation theorem.

Lemma B.1. The identity $F\left(\sigma_{z} \times 1\right)=y^{k / 2} f(z)$ for $z=x+i y \in \mathbb{H}$ induces a bijection $f \leftrightarrow F$ between the space of automorphic functions $f$ of weight $k$ on $\Gamma$ and the space of smooth functions $F: B_{\mathbb{A}}^{\times} \rightarrow \mathbb{C}$ satisfying $F\left(z \gamma g \kappa_{\infty} \kappa_{0}\right)=F(g) \chi_{k}\left(\kappa_{\infty}\right)$, where $z \in Z\left(B^{\times}\right)_{\mathbb{A}}, \gamma \in B_{\mathbb{Q}}^{\times}, g \in B_{\mathbb{A}}^{\times}, \kappa_{\infty} \in K_{\infty} \subset B_{\infty+}^{\times}, \kappa_{0} \in \hat{R}^{\times}$and $\chi_{k}: K_{\infty}=$ $\mathrm{SO}(2) \rightarrow S^{1}$ is given by $\left(\begin{array}{cc}\cos \theta & \sin \theta \\ -\sin \theta & \cos \theta\end{array}\right) \mapsto e^{i k \theta}$. If $f_{1} \leftrightarrow F_{1}$ and $f_{2} \leftrightarrow F_{2}$, then

$$
\begin{aligned}
\int_{\left[B^{1}\right]} \overline{F_{1}(g)} F_{2}(g) d^{(1)} g & =\frac{1}{2} \int_{[P B \times]} \overline{F_{1}(g)} F_{2}(g) d^{\times} g \\
& =\frac{1}{c(\Gamma)} \int_{\Gamma \backslash \mathbb{H}} y^{k} \overline{f_{1}(z)} f_{2}(z) \frac{d x d y}{y^{2}},
\end{aligned}
$$

where $c(\Gamma):=2 \pi^{-1} \zeta(2) d_{B} N \prod_{p \mid d_{B}}\left(1-p^{-1}\right) \prod_{p \mid N}\left(1+p^{-1}\right)$.

Remark B.2. One has $c(\Gamma)=\operatorname{vol}(\Gamma \backslash \mathbb{H})$ (see Remark 2.3$)$, or equivalently, $\operatorname{vol}\left(\left[B^{1}\right]\right)$ $=(1 / 2) \operatorname{vol}\left(\left[P B^{\times}\right]\right)=1$.

B.3. Weil representation. Let $B$ be a quaternion algebra over $\mathbb{Q}$. Let $\operatorname{GO}(B)$ be the orthogonal similitude group of the four-dimensional quadratic space $(B$, det), and $\nu: \mathrm{GO}(B) \rightarrow \mathbb{G}_{m}$ the similitude factor. Let $H=G\left(\mathrm{SL}_{2} \times \mathrm{O}(B)\right)=\{(g, h) \in$ $\left.\mathrm{GL}_{2} \times \mathrm{GO}(B): \operatorname{det}(g)=\nu(h)\right\}$. Then $H(\mathbb{A})$ acts on the space $\mathcal{S}(B(\mathbb{A}))=\otimes^{\prime} \mathcal{S}\left(B_{v}\right)$ 
of Schwarz-Bruhat functions via the Weil representation $\omega=\otimes \omega_{v}$, characterized by (see [19, §3])

$$
\begin{aligned}
\omega_{v}(n(x) t(y), 1) \varphi_{v}(\alpha) & =|t|_{v}^{4 / 2} \mathbf{e}_{v}(x \operatorname{det}(\alpha)) \varphi_{v}(t \alpha) \quad \text { for } x \in \mathbb{Q}_{v}, y \in \mathbb{Q}_{v}^{*}, \\
\omega_{v}(w, 1) \varphi_{v}(\alpha) & =(-1)^{\mathbf{1}_{v} \mid d_{B}} \mathcal{F} \varphi_{v}(\alpha), \\
\omega_{v}\left(\left[\begin{array}{rr}
1 & \\
& \nu(h)
\end{array}\right], h\right) \varphi_{v}(\alpha) & =|\nu(h)|_{v}^{-4 / 4} \varphi_{v}\left(h^{-1} \alpha\right) \quad \text { for } h \in \operatorname{GO}(B)_{v} .
\end{aligned}
$$

Here $\mathbf{1}_{v \mid d_{B}}$ is 1 if $v$ divides $d_{B}$ and 0 otherwise, while

$$
n(x):=\left[\begin{array}{ll}
1 & x \\
& 1
\end{array}\right], \quad t(y):=\left[\begin{array}{ll}
y & \\
& y^{-1}
\end{array}\right], \quad w:=\left[\begin{array}{rr}
1 \\
-1
\end{array}\right] .
$$

B.4. Theta correspondence. There is a morphism $\rho: B^{\times} \times B^{\times} \rightarrow \mathrm{GO}(B)$ given by $\rho\left(b_{1}, b_{2}\right)(\alpha)=b_{1} \alpha b_{2}^{-1}$, which surjects onto the connected component and satisfies $\operatorname{det}\left(b_{1} b_{2}^{-1}\right)=\nu\left(\rho\left(b_{1}, b_{2}\right)\right)$. For $\varphi \in \mathcal{S}\left(B_{\mathbb{A}}\right), g \in \mathrm{GL}_{2}(\mathbb{A})$ and $\left(b_{1}, b_{2}\right) \in\left(B^{\times} \times B^{\times}\right)(\mathbb{A})$ with $\operatorname{det}(g)=\operatorname{det}\left(b_{1} b_{2}^{-1}\right)$, define the theta kernel

$$
\theta_{\varphi}\left(g ; b_{1}, b_{2}\right)=\sum_{\alpha \in B} \omega\left(g, \rho\left(b_{1}, b_{2}\right)\right) \varphi(\alpha) .
$$

For fixed $b_{1}, b_{2} \in B^{\times}(\mathbb{A})$ and $g^{\prime} \in \mathrm{GL}_{2}(\mathbb{A})$ with $\operatorname{det}\left(g^{\prime}\right)=\operatorname{det}\left(b_{1} b_{2}^{-1}\right)$, the function $\mathrm{SL}_{2}(\mathbb{A}) \ni g \mapsto \theta_{\varphi}\left(g g^{\prime} ; b_{1}, b_{2}\right)$ is left-SL ${ }_{2}(\mathbb{Q})$-invariant. If $F$ is a function on $\mathrm{GL}_{2}(\mathbb{Q}) \backslash \mathrm{GL}_{2}(\mathbb{A})$ of rapid decay modulo the center, define for $b_{1}, b_{2} \in B^{\times}(\mathbb{A})$ its theta lift

$$
\Theta_{\varphi}(\bar{F})\left(b_{1}, b_{2}\right)=\int_{g \in\left[\mathrm{SL}_{2}\right]} \bar{F}\left(g g^{\prime}\right) \theta_{\varphi}\left(g g^{\prime} ; b_{1}, b_{2}\right) d^{(1)} g,
$$

where $g^{\prime} \in \mathrm{GL}_{2}(\mathbb{A})$ is any element for which $\operatorname{det}\left(g^{\prime}\right)=\operatorname{det}\left(b_{1} b_{2}^{-1}\right)$.

B.5. The precise lifting for newforms. Let notation be as in \$5.1. Let $f_{B} \leftrightarrow F_{B}$ and $f \leftrightarrow F$ via Lemma B.1. Define $\varphi=\otimes \varphi_{v} \in \mathcal{S}(B(\mathbb{A}))$ via $\varphi_{p}=\operatorname{vol}\left(R_{p}^{\times}, d^{\times} x\right)^{-1} \mathbf{1}_{R_{p}}$ and $\varphi_{\infty}=\pi^{-1} X^{k} e^{-2 \pi P}$, where $\mathbf{1}_{R_{p}}$ is the characteristic function of $R_{p}$, and $X$ and $P$ are as in 3.2. This choice is consistent with [39, §2.3]. The definitions imply:

Lemma B.3. For $z, z_{1}, z_{2} \in \mathbb{H}$, we have $\theta_{\varphi}\left(\sigma_{z} ; \sigma_{z_{1}}, \sigma_{z_{2}}\right)=c\left(\Gamma^{\prime}\right) y^{k / 2} \theta_{z_{1}, z_{2}}(z)$.

Remark B.4. To get the Fourier expansion for $\theta_{z_{1}, z_{2}} \mid \tau_{d}(z)$ claimed in Appendix A.1 we use that $c\left(\Gamma^{\prime}\right) y^{k / 2} \theta_{z_{1}, z_{2}} \mid \tau_{d}(z)=\theta_{\varphi}\left(\tau_{d} \sigma_{z} \times 1 ; \sigma_{z_{1}}, \sigma_{z_{2}}\right)=\theta_{\varphi}\left(\sigma_{z} \times \tau_{d}^{-1} ; \sigma_{z_{1}}, \sigma_{z_{2}}\right)$ and apply the definition of the Weil representation at the finite places. The key calculation is that at a prime $p$ for which we may identify $B_{p}=M_{2}\left(\mathbb{Q}_{p}\right)$, we have

$$
\mathcal{F} 1\left(\begin{array}{cc}
\mathbb{Z}_{p} & \mathbb{Z}_{p} \\
p^{\alpha} \mathbb{Z}_{p} & \mathbb{Z}_{p}
\end{array}\right)=p^{-\alpha} 1\left(\begin{array}{cc}
\mathbb{Z}_{p} p^{-\alpha} \mathbb{Z}_{p} \\
\mathbb{Z}_{p} & \mathbb{Z}_{p}
\end{array}\right) \cdot
$$

We omit the details, since they are discussed in [26] and [39, §2.1].

Theorem B.5. For $b_{1}, b_{2} \in B^{\times}(\mathbb{A})$, we have $\overline{F_{B}}\left(b_{1}\right) F_{B}\left(b_{2}\right)=\frac{\left\|F_{B}\right\|^{2}}{\|F\|^{2}} \Theta_{\varphi}(\bar{F})\left(b_{1}, b_{2}\right)$, where

$$
\left\|F_{B}\right\|^{2}:=\frac{1}{2} \int_{\left[P B^{\times}\right]}\left|F_{B}\right|^{2}(g) d^{\times} g, \quad\|F\|^{2}:=\frac{1}{2} \int_{\left[\mathrm{PGL}_{2}\right]}|F|^{2}(g) d^{\times} g .
$$

Proof. This is [39, Thm 1]. Although Watson assumes throughout his paper that $N$ is squarefree, his proof of this particular result applies verbatim for general $N$. 
Proof of Theorem 5.2. By Lemma B.1, Lemma B.3 and Theorem B.5.

$$
\begin{aligned}
\left(y_{1} y_{2}\right)^{k} \overline{f_{B}\left(z_{1}\right)} f_{B}\left(z_{2}\right) & =\overline{F_{B}\left(\sigma_{z_{1}}\right)} F_{B}\left(\sigma_{z_{2}}\right)=\frac{\left\|F_{B}\right\|^{2}}{\|F\|^{2}} \Theta_{\varphi}(\bar{F})\left(\sigma_{z_{1}}, \sigma_{z_{2}}\right) \\
& =\frac{\left\|F_{B}\right\|^{2}}{\|F\|^{2}} \int_{\left[\mathrm{SL}_{2}\right]} \bar{F}(g) \theta_{\varphi}\left(g ; \sigma_{z_{1}}, \sigma_{z_{2}}\right) d^{(1)} g \\
& =\frac{\left\|F_{B}\right\|^{2}}{\|F\|^{2}} \frac{1}{c(\Gamma)} \int_{\Gamma \backslash \mathbb{H}} y^{k / 2} \overline{f(z)} \theta_{\varphi}\left(\sigma_{z} ; \sigma_{z_{1}}, \sigma_{z_{2}}\right) \frac{d x d y}{y^{2}} \\
& =C \int_{\Gamma \backslash \mathbb{H}} y^{k} \overline{f(z)} \theta_{z_{1}, z_{2}}(z) \frac{d x d y}{y^{2}}, \quad C:=\frac{\left\|F_{B}\right\|^{2}}{\|F\|^{2}} \frac{c\left(\Gamma^{\prime}\right)}{c(\Gamma)}
\end{aligned}
$$

Since $f_{B}$ and $f$ are compatibly normalized (see Definition 2.2), we have $C=1$.

\section{ACKNOWLEDGMENTS}

The problem considered in this paper, as well as our general strategy, was conceived over the course of several fruitful conversations with Kartik Prasanna; it is a pleasure to thank him for numerous helpful discussions that have contributed substantially to our own understanding. The authors thank Andrew Snowden for helpful discussions during a visit to the University of Michigan in January 2011, at which the first computations of the sort discussed in this paper were carried out. The author also thanks Henri Darmon, Daniel Disegni, Jon Hanke, Eren Kiral Mehmet, Victor Rotger, William Stein, Carlos de Vera, and John Voight for helpful discussions and encouragement during the Arizona Winter School 2011, of which we thank the organizers for their support. We thank Nahid Walji for his helpful feedback on an earlier draft of this paper.

\section{REFERENCES}

[1] A. O. L. Atkin and J. Lehner, Hecke operators on $\Gamma_{0}(m)$, Math. Ann. 185 (1970), 134-160. MR0268123(42 \#3022)

[2] A. R. Booker, A. Strömbergsson, and A. Venkatesh, Effective computation of Maass cusp forms, Int. Math. Res. Not., posted on 2006, Art. ID 71281, 34, DOI 10.1155/IMRN/2006/71281. MR2249995 (2007i:11073)

[3] H. Carayol, Sur la mauvaise réduction des courbes de Shimura (French), Compositio Math. 59 (1986), no. 2, 151-230. MR860139 (88a:11058)

[4] H. Darmon and R. Pollack, Efficient calculation of Stark-Heegner points via overconvergent modular symbols, Israel J. Math. 153 (2006), 319-354, DOI 10.1007/BF02771789. MR:2254648 (2007k:11077)

[5] Henri Darmon and Victor Rotger, Algebraic cycles and Stark-Heegner points, http:// www-ma2 .upc.edu/vrotger/docs/AWS2011/aws.pdf, 2011.

[6] L. Dembélé, Quaternionic Manin symbols, Brandt matrices, and Hilbert modular forms, Math. Comp. 76 (2007), no. 258, 1039-1057, DOI 10.1090/S0025-5718-06-01914-4. MR2291849 (2008g:11078)

[7] L. Dembélé and S. Donnelly, Computing Hilbert modular forms over fields with nontrivial class group, Algorithmic number theory, Lecture Notes in Comput. Sci., vol. 5011, Springer, Berlin, 2008, pp. 371-386, DOI 10.1007/978-3-540-79456-1_25. MR2467859(2010d:11149)

[8] N. D. Elkies, Heegner point computations, Algorithmic Number Theory (Ithaca, NY, 1994), Lecture Notes in Comput. Sci., vol. 877, Springer, Berlin, 1994, pp. 122-133, DOI 10.1007/3540-58691-1_49. MR1322717 (96f:11080)

[9] N. D. Elkies, Shimura curve computations, Algorithmic Number Theory (Portland, OR, 1998), Lecture Notes in Comput. Sci., vol. 1423, Springer, Berlin, 1998, pp. 1-47, DOI 10.1007/BFb0054850. MR.1726059 (2001a:11099) 
[10] D. Goldfeld, The Gauss class number problem for imaginary quadratic fields, Heegner Points and Rankin L-Series, Math. Sci. Res. Inst. Publ., vol. 49, Cambridge Univ. Press, Cambridge, 2004, pp. 25-36, DOI 10.1017/CBO9780511756375.004. MR2083209 (2005f:11251)

[11] I. S. Gradshteyn and I. M. Ryzhik, Table of Integrals, Series, and Products, 7th ed., Elsevier/Academic Press, Amsterdam, 2007. Translated from the Russian; Translation edited and with a preface by Alan Jeffrey and Daniel Zwillinger; With one CD-ROM (Windows, Macintosh and UNIX). MR2360010 (2008g:00005)

[12] M. Greenberg, Heegner point computations via numerical p-adic integration, Algorithmic Number Theory, Lecture Notes in Comput. Sci., vol. 4076, Springer, Berlin, 2006, pp. 361376, DOI 10.1007/11792086_26. MR2282936 (2008a:11069)

[13] M. Greenberg, Heegner Points and Rigid Analytic Modular Forms, ProQuest LLC, Ann Arbor, MI, 2006. Thesis (Ph.D.)-McGill University (Canada). MR2710023

[14] M. Greenberg and J. Voight, Computing systems of Hecke eigenvalues associated to Hilbert modular forms, Math. Comp. 80 (2011), no. 274, 1071-1092, DOI 10.1090/S0025-5718-201002423-8. MR2772112 (2012c:11103)

[15] B. H. Gross and D. B. Zagier, Heegner points and derivatives of L-series, Invent. Math. 84 (1986), no. 2, 225-320, DOI 10.1007/BF01388809. MR833192 (87j:11057)

[16] B. H. Gross, Heegner points on $X_{0}(N)$, Modular Forms (Durham, 1983), Ellis Horwood Ser. Math. Appl.: Statist. Oper. Res., Horwood, Chichester, 1984, pp. 87-105. MR803364 (87f:11036b)

[17] B. H. Gross, Kolyvagin's work on modular elliptic curves, L-Functions and Arithmetic (Durham, 1989), London Math. Soc. Lecture Note Ser., vol. 153, Cambridge Univ. Press, Cambridge, 1991, pp. 235-256, DOI 10.1017/CBO9780511526053.009. MR.1110395 (93c:11039)

[18] P. E. Gunnells and D. Yasaki, Hecke operators and Hilbert modular forms, Algorithmic Number Theory, Lecture Notes in Comput. Sci., vol. 5011, Springer, Berlin, 2008, pp. 387-401, DOI 10.1007/978-3-540-79456-1_26. MR2467860 (2010a:11081)

[19] M. Harris and S. S. Kudla, The central critical value of a triple product L-function, Ann. of Math. (2) 133 (1991), no. 3, 605-672, DOI 10.2307/2944321. MR1109355 (93a:11043)

[20] R. Holowinsky, Sieving for mass equidistribution, Ann. of Math. (2) 172 (2010), no. 2, 14991516. MR2680498 (2011i:11060)

[21] H. Iwaniec, Spectral Methods of Automorphic Forms, 2nd ed., Graduate Studies in Mathematics, vol. 53, American Mathematical Society, Providence, RI, 2002. MR 1942691|(2003k:11085)

[22] H. Iwaniec and E. Kowalski, Analytic Number Theory, American Mathematical Society Colloquium Publications, vol. 53, American Mathematical Society, Providence, RI, 2004. MR2061214(2005h:11005)

[23] D. Jetchev, K. Lauter, and W. Stein, Explicit Heegner points: Kolyvagin's conjecture and non-trivial elements in the Shafarevich-Tate group, J. Number Theory 129 (2009), no. 2, 284-302, DOI 10.1016/j.jnt.2008.05.007. MR2473878 (2009m:11080)

[24] D. R. Kohel and H. A. Verrill, Fundamental domains for Shimura curves (English, with English and French summaries), J. Théor. Nombres Bordeaux 15 (2003), no. 1, 205-222. Les XXIIèmes Journées Arithmetiques (Lille, 2001). MR2019012 (2004k:11096)

[25] P. D. Nelson, A. Pitale, and A. Saha, Bounds for Rankin-Selberg integrals and quantum unique ergodicity for powerful levels, ArXiv e-prints, May 2012.

[26] P. D. Nelson, Computing on Shimura curves II: Implementing the Shimizu lifting, Appendix $\mathrm{C}$ of the notes Periods and special values of L-functions, http://swc.math.arizona.edu/ aws/2011/2011PrasannaNotesProject.pdf, 2011.

[27] A. Pizer, An algorithm for computing modular forms on $\Gamma_{0}(N)$, J. Algebra 64 (1980), no. 2, 340-390, DOI 10.1016/0021-8693(80)90151-9. MR579066 (83g:10020)

[28] K. Prasanna, Computing on Shimura curves I: Expansions at CM points, Appendix B of the notes Periods and special values of L-functions, http://swc.math.arizona.edu/aws/2011/ 2011PrasannaNotesProject.pdf, 2011.

[29] H. Shimizu, On zeta functions of quaternion algebras, Ann. of Math. (2) 81 (1965), 166-193. MR.0171771 (30 \#1998)

[30] H. Shimizu, Theta series and automorphic forms on $\mathrm{GL}_{2}$, J. Math. Soc. Japan 24 (1972), 638-683. MR0333081 (48 \#11406) 
[31] G. Shimura, Introduction to the Arithmetic Theory of Automorphic Functions, Publications of the Mathematical Society of Japan, No. 11. Iwanami Shoten, Publishers, Tokyo, 1971. Kanô Memorial Lectures, No. 1. MR0314766 (47 \#3318)

[32] W. Stein, Modular Forms, a Computational Approach, Graduate Studies in Mathematics, vol. 79, American Mathematical Society, Providence, RI, 2007. With an appendix by P. E. Gunnells. MR:2289048(2008d:11037)

[33] W. A. Stein et al., Sage Mathematics Software (Version 4.1.1), The Sage Development Team, 2009. http://www. sagemath.org.

[34] M.-F. Vignéras, Arithmétique des Algèbres de Quaternions (French), Lecture Notes in Mathematics, vol. 800, Springer, Berlin, 1980. MR.580949 (82i:12016)

[35] J. Voight and J. Willis, Computing power series expansions of modular forms, ArXiv e-prints, April 2012.

[36] J. Voight, Computing CM points on Shimura curves arising from cocompact arithmetic triangle groups, Algorithmic Number Theory, Lecture Notes in Comput. Sci., vol. 4076, Springer, Berlin, 2006, pp. 406-420, DOI 10.1007/11792086_29. MR2282939 (2008g:11104)

[37] J. Voight, Computing fundamental domains for Fuchsian groups (English, with English and French summaries), J. Théor. Nombres Bordeaux 21 (2009), no. 2, 469-491. MR2541438 (2011c:11064)

[38] J. Voight, Computing automorphic forms on Shimura curves over fields with arbitrary class number, Algorithmic Number Theory, Lecture Notes in Comput. Sci., vol. 6197, Springer, Berlin, 2010, pp. 357-371, DOI 10.1007/978-3-642-14518-6_28. MR2721432(2011m:11098)

[39] T. C. Watson, Rankin Triple Products and Quantum Chaos, ProQuest LLC, Ann Arbor, MI, 2002. Thesis (Ph.D.)-Princeton University. MR.2703041

[40] S. Zhang, Heights of Heegner points on Shimura curves, Ann. of Math. (2) 153 (2001), no. 1, 27-147, DOI 10.2307/2661372. MR.1826411 (2002g:11081)

EPFL, Station 8, CH-1015 Lausanne, Switzerland

Current address: ETH Zurich, Raemistrasse 101, 8092 Zurich, Switzerland

E-mail address: Paul.nelson@math.ethz.ch 